\title{
Induction Heating of Thin Strips in Transverse Flux Magnetic Field
}

\author{
Jerzy Barglik \\ Silesian University of Technology \\ Poland
}

\section{Introduction}

An idea of transverse flux induction heating has been well known for more than sixty years. Some of its main principles were presented for instance by Russian physicist Volovgin (Mühlbauer, 2008). Many papers on the topic considering mainly theoretical aspects of the task were published in the last sixty years (Baker, 1950), (Jackson, 1972), (Barglik, 1992), (Mühlbauer et al, 1995), (Tudorache \& Fireteanu, 1998), (Nacke et al, 2001), (Dughiero et al, 2003). Usage of transverse flux induction heating system have been effective in case of thin strips of the thickness comparable with the depth of electromagnetic field penetration $\delta$. It makes it possible to obtain required parameters of the process like: uniformity of temperature distribution within the workpiece and big total energy efficiency at rather low frequencies of the field current (in many cases also for mains frequency $f=50$ or $60 \mathrm{~Hz}$ ) in comparison with more often used classical induction heaters working with longitudinal magnetic field. The chapter deals with continual transverse flux induction heating of thin non-ferrous metal strips. The three-dimensional model used for the analysis was based on a system of non-linear differential equations for coupled electromagnetic and temperature fields that were solved by numerical methods. Development of the mathematical modelling of transverse flux induction heating as well as the computations by means of professional software and supplementary user codes reach quite a high level. But there is still a problem with the accuracy of calculations that was associated mainly with reliability of existing mathematical and numerical models with respect to the physical reality. The reason consists in various simplifications accepted for shortening the time of calculations and level of knowledge of temperature dependent material properties as well as convection and radiation heat transfer coefficients for the particular task. So the best way for obtaining data necessary for an optimal design and construction of induction heaters seems to be a numerical analysis supported by well planned experiments. Illustrative examples of continual transverse flux induction heating were analyzed. Results of numerical simulation were presented and compared with measurement data taken from laboratory stands. Quite good accordance between calculations and measurements was achieved. An illustrative example demonstrates that one of the ways making possible to obtain an uniform temperature distribution along the width of the workpiece could be a proper selection of suitable frequency of the field current. 


\section{Idea of transverse flux induction heating}

Induction heating of thin flat workpieces, similar like levitation or cold crucible melting, induction heating for semi-liquid state before plastic working and surface induction hardening belongs to the quickest developed areas of modern electroheat (Barglik, 2010), (Baake \& Nacke, 2010). Usage of typical induction heaters with longitudinal magnetic field do not allow to obtain high enough electrical efficiency of inductor-workpiece system. In order to achieve high enough value of electrical efficiency for induction heating system with longitudinal magnetic field the ratio of workpiece thickness $g$ to electromagnetic field penetration $\delta$ should be equal or bigger than 3.5

$$
g \geq 3.5 \delta
$$

However it requires to use a field current with properly increased frequency and consequently necessity of an expensive power source. Usage of induction heater with transverse flux field makes it possible to obtain big enough electrical efficiency of inductorworkpiece system at distinctly lower frequency of field current. So it is possible to use in that case cheaper power source. Transverse flux magnetic field penetrates the workpiece and has practically only normal component of magnetic flux density perpendicular to external plane of the flat workpiece. The tangent component of magnetic flux density is more and more smaller and often could be neglected. Figure 1 presents comparison of electrical efficiency of inductor-workpiece systems with transverse flux and longitudinal magnetic fields. It shows dependencies of electrical efficiency for both systems in case of stationary heating of brass strip with thickness of $3.2 \mathrm{~mm}$.

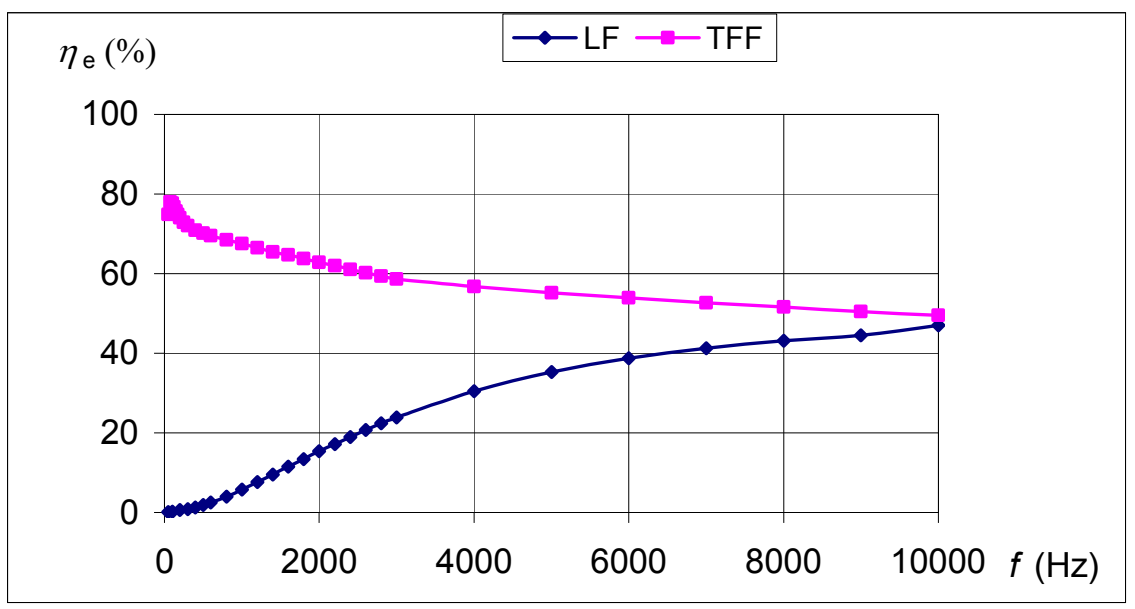

Fig. 1. Comparison of electrical efficiency of induction heater with longitudinal (LF) and transverse flux (TFF) fields on field current frequency (Barglik, 2005)

Two-dimensional and three dimensional models making possible to determine a distribution of electromagnetic and temperature fields in conductive workpiece were elaborated. Some kind of summary was presented for instance in (Barglik, 2002). It contains a broad overview of earlier presented papers on application of transfer flux induction 
heating systems. A special emphasis was put on flexible transverse flux induction heating systems for non-ferrous workpieces, making possible to heat strips with different widths by means of universal type of inductor. In order to solve such a problem the inductor could be divided into a few single inductors with different widths and also various positions to the axis of the strip. Usage of transverse flux induction heating systems is especially effective in case of heating of strips of thickness comparable with electromagnetic field penetration. It makes it possible to achieve expected process parameters like uniformity of temperature distribution in a whole volume of the workpiece and big total efficiency of the heater at relatively low field current frequency (sometimes even at mains frequency $f=50$ or $60 \mathrm{~Hz}$ ) in comparison with much bigger values typically obtained for classical longitudinal induction heating systems. Three-dimensional calculation model based upon partial differential equations for electromagnetic and temperature fields was elaborated and solved by proper numerical methods. Development of mathematical methods of tranverse flux induction heating systems and used professional software, numerical methods have reached a satisfied big level. A kind of problem seems to be an accuracy of calculations connected mainly with necessary simplifications of mathematical models making possible shortening of time of calculations and uncertainty of material properties of their temperature dependencies (Barglik, 2000). The best way for obtaining of data for calculation and designing of any transverse flux induction heating systems seems to be numerical analysis supplemented by well planned experimental part. Continual induction heating of thin nonferrous metals strips (copper, brass, aluminum) was presented in the paper. Illustrative examples describing continual induction heating were described and analyzed. Results of simulation were compared with measurements done at laboratory stands.

\section{Induction annealing}

Term induction annealing means softening of treated material with usage induction heating (Davies, 1990). The process consists of rapid induction heating of treated material to a particular temperature, keeping it through some period of time and then cooling with a selected velocity. As a result requested microstructure and suspected softness of the material was obtained. Scheme of the industrial technologicial line for continual annealing of copper and its alloys strips in a classical horizontal system and a modified annealing line applied initial induction heating were presented in Fig. 2. Upper figure 2 presents a classical line. Strip (2) moves from uncoiler drum (1) through two zones of the technological line: heating chamber (3) and cooling chamber (5) to the final drum (6). In order to protect the strip against oxidation any protective atmosphere was used. In order to achieve requested velocity of strip movement of about $0.5 \mathrm{~m} / \mathrm{s}$ it is necessary to use a long heating zone. It causes that the total length of the device often could be longer than $30 \mathrm{~m}$. Lower figure 2 shows modified annealing line. Strip (2) moves from uncoiler drum (1) to a zone of induction heating. The induction heater has created a chamber jointly with resistance device (the induction-resistance device (7)). At the end of the induction heater zone the strip has reached the requested final temperature with uniform distribution within the annealed material. Resistance part of the device (7) makes it possible keeping of constant temperature for requested period of time. It is possible to use instead of indirect resistance device another electroheat device like for instance infrared heater (Hering, 1992). 

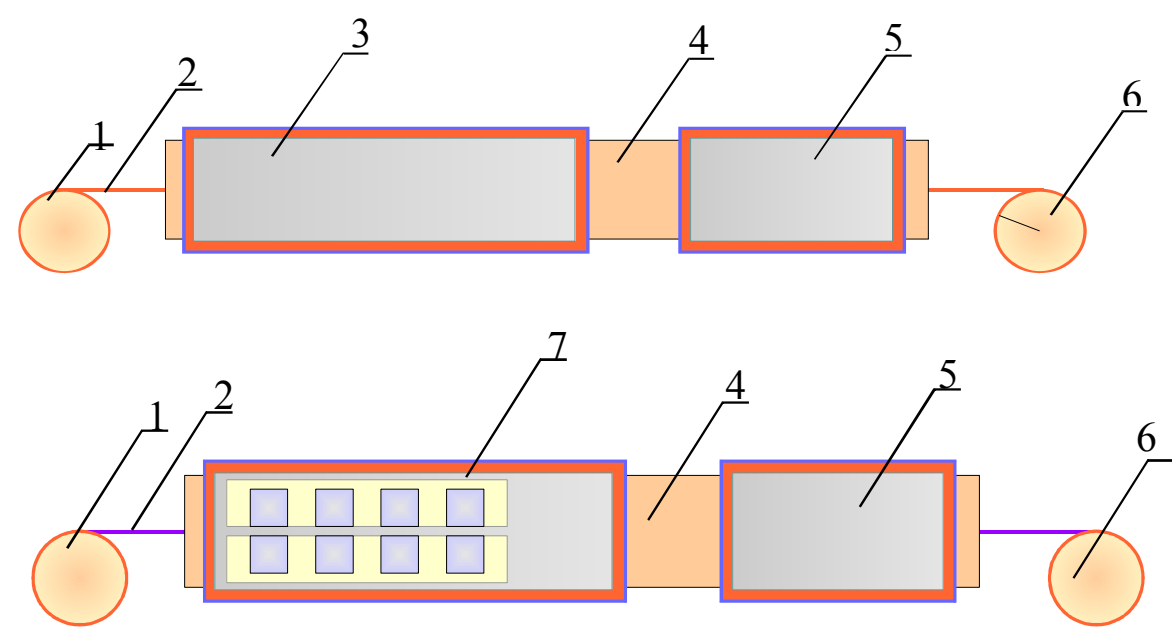

Fig. 2. Block scheme of technological annealing line in a horizontal system: classical line (upper figure), modified line with induction heating (lower figure). 1 - uncoiler drum, 2 - non-ferrous strip, 3 - heating chamber, 4 - container with inert atmosphere, 5 - cooling chamber, 6 - final drum, 7 - induction- resistance device.

Based upon exploitation experiences of the technological lines for annealing of copper and brass strips with initial transverse flux induction heating in Poland and abroad it has been possible to confirm that due to the modification total energy consumption has decreased for about $30 \%$. A different solution could be a vertical technological line. Besides of clear advantages of such a solution there was also some disadvantages (Barglik, 2000).

\section{Basic of transverse flux induction heating}

Let us consider transverse flux induction heating system used for instance for low temperature heating of thin non-ferrous metals strips (copper, brass, aluminum, lead and their alloys) (Fig.3). The moving strip (1) was placed symetrically in a narrow air-gap of two-side multipolar inductor. The strip moves with a constant velocity in $x$ direction. Winding (2) was placed in grooves of the laminated magnetic core (3). It was constructed from copper profile conductors cooled by water. Coils were connected in series. The transverse flux induction heating system was supplied by one-phase harmonic current of frequency $f$ and effective value of current $I_{z}$. As a power source directly power network or more frequently thyristor convertor were used. Usage of thyristor power source makes possible easy regulation of both quantities frequency of field current and power. Sometimes transistor power sources were used. A way of inductor connection and consequent direction of field current was indicated in Fig. 3 by red arrows. For such a system of connections in the moving strip time-varying transverse flux magnetic field was generated. Of course, if we modify a way of winding connection we could arrange a classical induction heating system with the longitudinal magnetic field (Barglik, 2002). A view of such an universal inductor was shown in Fig. 4. 


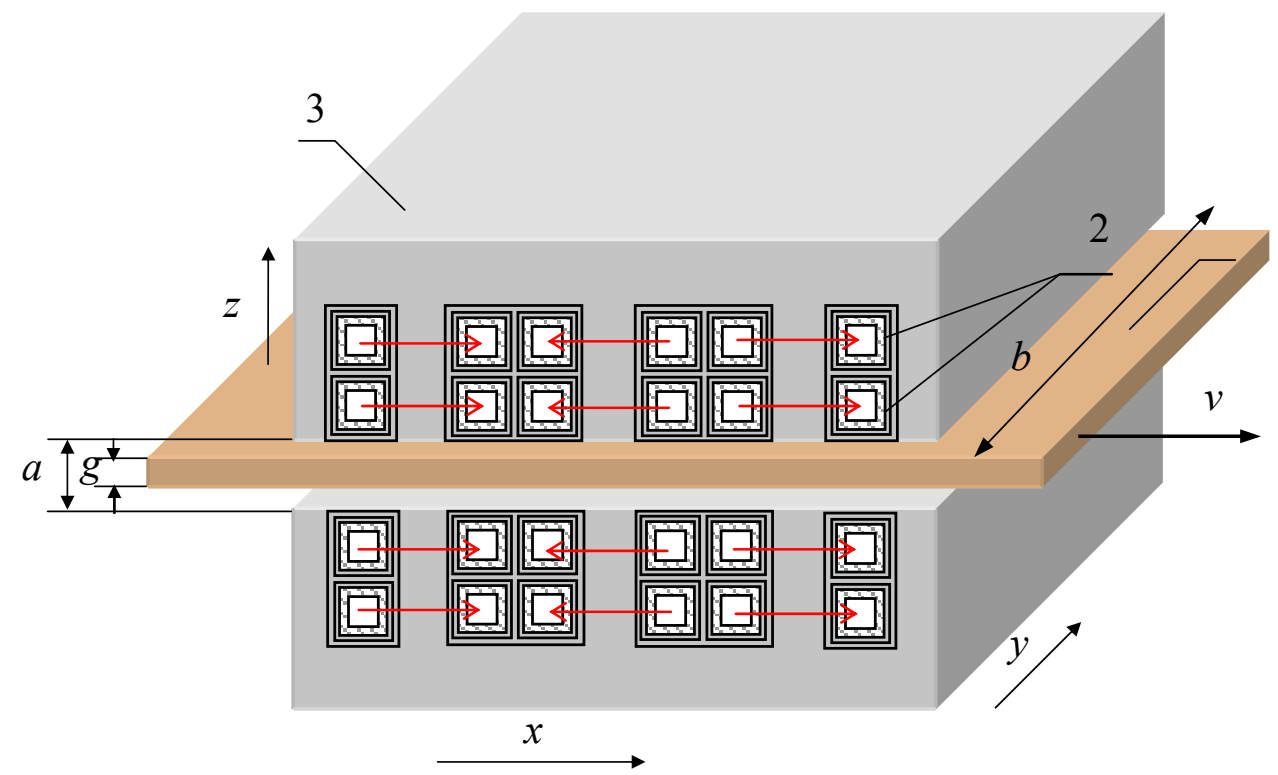

Fig. 3. Scheme of TFIH system. 1 - strip, 2 - winding, 3 - magnetic core, $a$ - air-gap, $b$ - width of the strip, $g$ - thickness of the strip. Red arrows show current direction in winding

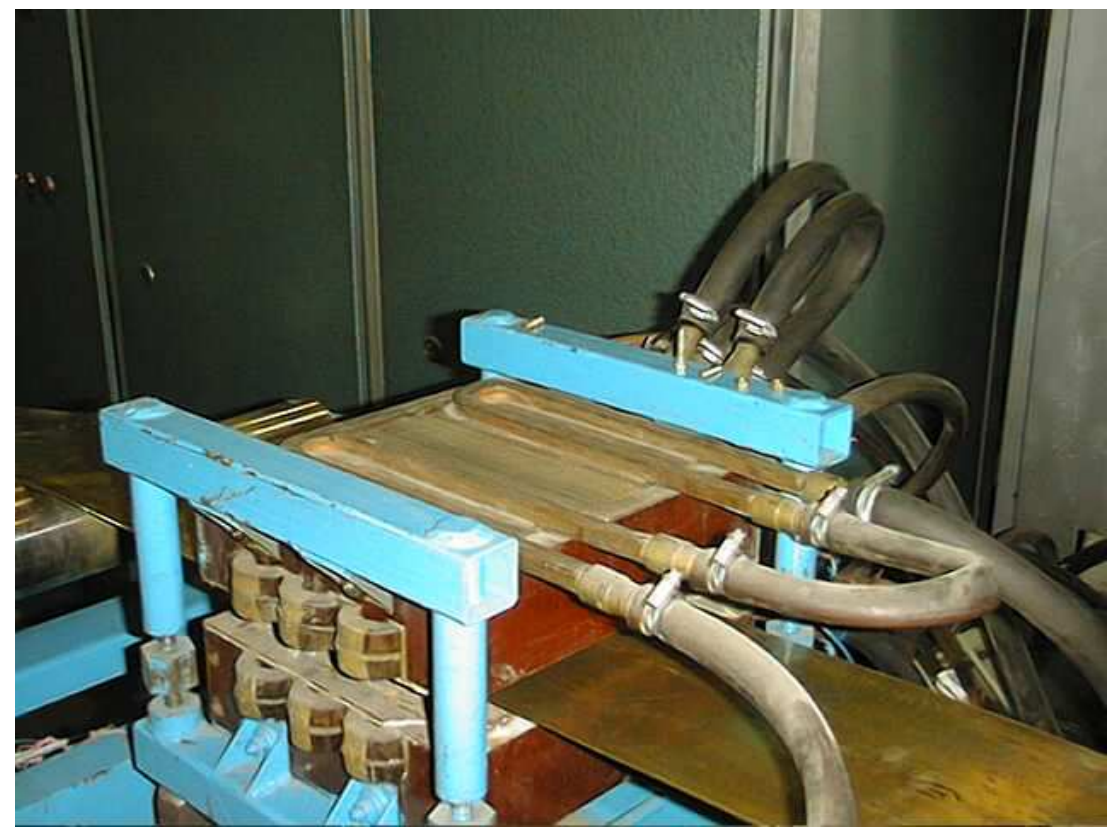

Fig. 4. A view of inductor from Fig.3 


\section{Mathematical model}

Mathematical model of such multiphysic problem is quite complicated because of interaction between electromagnetic and temperature fields. In many cases it is also necessary to take into consideration also other physical fields like for instance heat stress field. The most important task is to recognize such parameters of the system like: frequency of field current, velocity of strip movement and its dimensions in order to obtain required exploitation parameters like: average value of workpiece temperature, uniformity of its distribution and big value of electrical efficiency of the system. Block scheme of calculation algorithm was shown in Fig. 5.

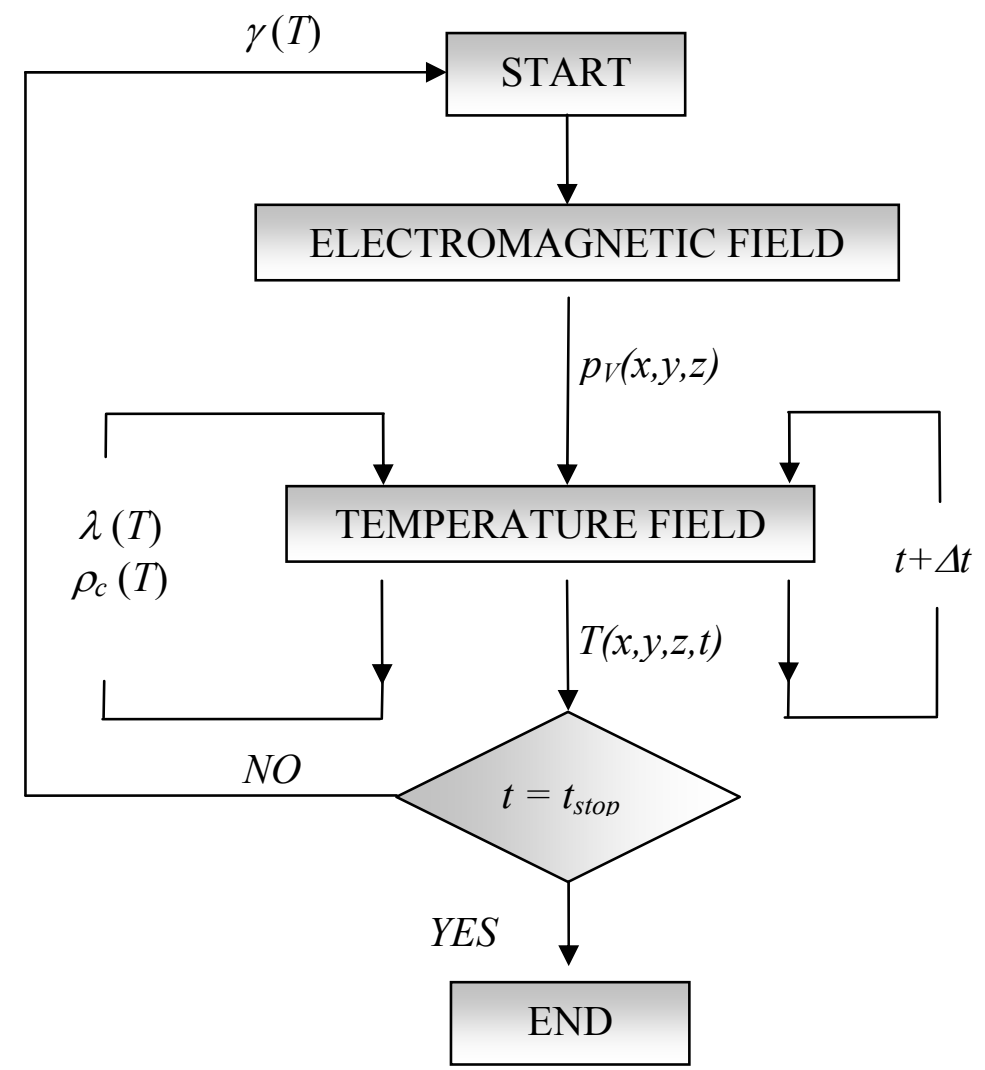

Fig. 5. Algorithm for calculation of coupled electromagnetic and temperature field

Calculations begin with the determination of electromagnetic field distribution in a whole area. Based upon that specific Joule losses released in the workpiece were calculated. Then temperature field was achieved. During these calculations non-linear dependencies of material properties on temperature were taken into consideration. If changes of the strip temperature were bigger than $\Delta T$ proper correction of electric conductivity were done and we start again with the electromagnetic calculations. The algorithm stops, if the proper selected criterion was satisfied. It was assumed that the criterion was satisfied for $t=t_{\text {stop }}$ 
Three-dimensional model for calculation of quasi-stationary electromagnetic field was shown in Fig. 6.
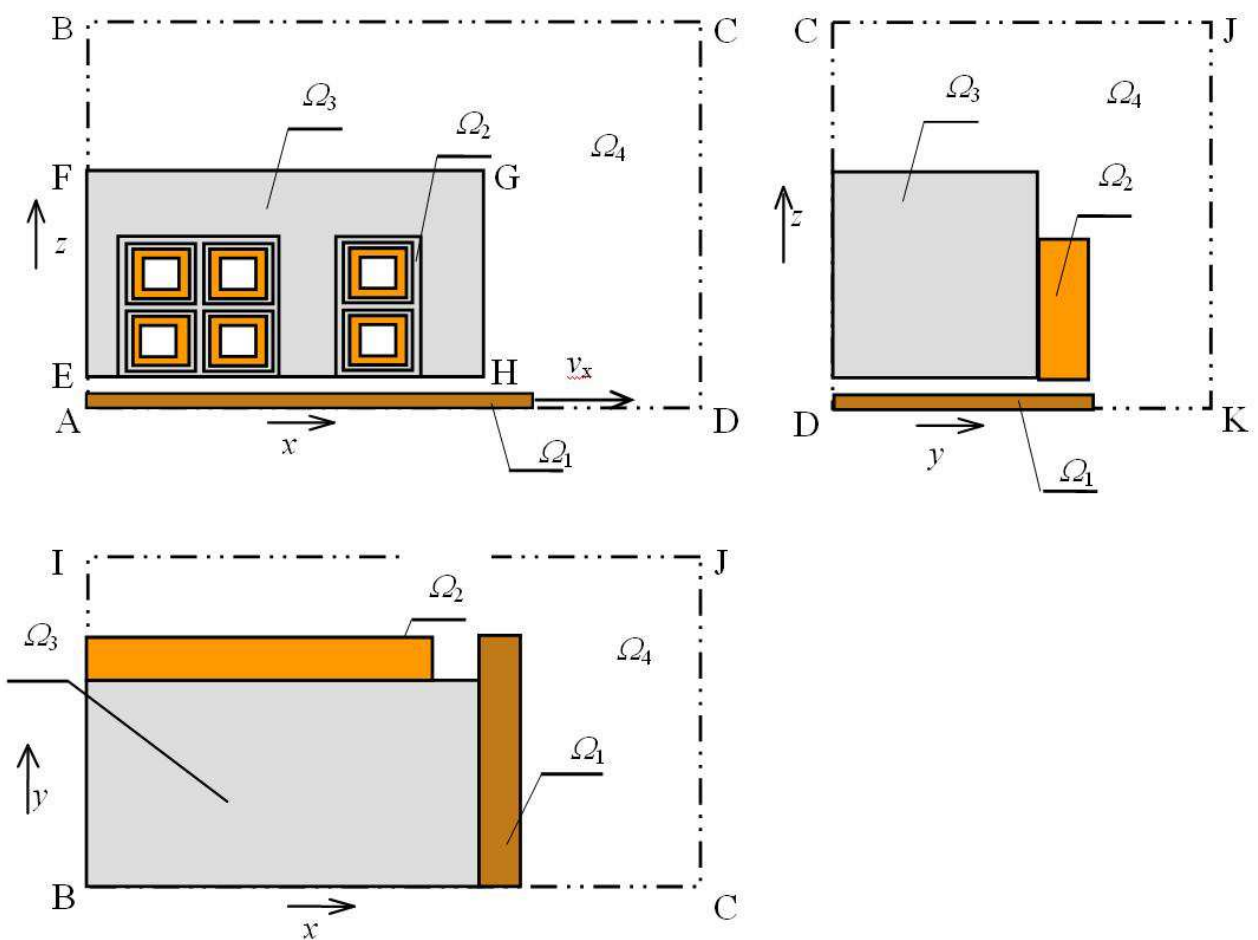

Fig. 6. Three-dimensional calculation model of electromagnetic field distribution.

Subdomains: $\Omega_{1}$ - strip, $\Omega_{2}$ - winding, $\Omega_{3}$ - magnetic core, $\Omega_{4}$ - surroundings (air),

Calculations were provided only for $1 / 8$ part of the total calculation domain taking into account symmetry conditions at planes $x=0, y=0, z=0$ (see Fig. 6) (Barglik, 2002). The task being in fact a kind of the open problem could be treated as a close one. It means that all the system was put to an interior part of rectangular prism which walls being external borders of the system were taken far enough to the inductor-workpiece arrangement. The borders were depicted in Fig. 6 by broken lines. Based upon testing calculations dimensions of the rectangular prism were determined. It was assumed that $\mathrm{AB}=7 \mathrm{AF}$ and $\mathrm{AD}=5.5 \mathrm{AH}$ (see Fig. 6). Electromagnetic field was described in a classical way by means of magnetic vector potential $A$.

$$
\operatorname{curl} \frac{1}{\mu} \operatorname{curl} \boldsymbol{A}+\gamma \frac{\partial \boldsymbol{A}}{\partial t}-\gamma(\boldsymbol{v} \times \operatorname{curl} \boldsymbol{A})=\boldsymbol{J}_{\mathrm{z}}
$$

where $\mu$ denotes magnetic permeability, $\gamma$ - electric conductivity, $v$ - velocity, $\boldsymbol{J}_{\mathrm{z}}$ - vector of field current density.

In case when magnetic permeability of magnetic core (subdomain $\Omega_{3}$ ) could be considered as constant equation (2) transforms to a following form for phasor of magnetic vector potential $\underline{A}$ 


$$
\operatorname{curlcurl} \underline{\boldsymbol{A}}+\mathrm{j} \omega \mu \gamma \underline{\boldsymbol{A}}-\mu \gamma(\boldsymbol{v} \times \operatorname{curl} \underline{\boldsymbol{A}})=\mu \underline{\boldsymbol{J}}_{z}
$$

where $\mathrm{j}$ denotes imaginary unit, $\omega$ - angular frequency.

For each subdomain equation (3) was modified by putting suitable values of angular frequency $\omega$, magnetic permeability $\mu$, electric conductivity $\gamma$, velocity of strip movement $v$ and field current density $\underline{\boldsymbol{J}}_{z}$ For instance for the subdomain $\Omega_{1}$ (strip moving in direction of $\mathrm{x}$ axis with velocity $v_{\mathrm{x}}$ ) equation (3) was transformed to a following form:

$$
\operatorname{curlcurl} \underline{\boldsymbol{A}}+\mathrm{j} \omega \mu \gamma \underline{\boldsymbol{A}}-\mu \gamma\left(v_{x}, 0,0\right) \times \operatorname{curl} \underline{\boldsymbol{A}}=0
$$

Equation (3) could be additionally simplified by neglecting of the third component of the left side. It could be done for not big values of strip velocity. In order to check what kind of unaccuracy could be introduce as a result of such a simplification additional calculations were provided making possible to compare specific Joule losses caused by movement of the strip $p_{\mathrm{vm}}$ with specific Joule losses caused by eddy currents induced in the strip by timevarying electromagnetic field $p_{\mathrm{vi}}$. Let us consider a ratio of these two values of specific Joulle losses as velocity coefficient $k_{\mathrm{r}}$.

$$
k_{\mathrm{r}}=\frac{\int_{V} p_{\mathrm{vr}} \mathrm{d} V}{\int_{V} p_{\mathrm{vi}} \mathrm{d} V}
$$

Calculations were done for two different frequencies of field current $(f=50,2000 \mathrm{~Hz})$ and for several velocities of strip movement. Exemplary results showing dependence of velocity coefficient on velocity of strip movement were presented in Fig. 7. For mains frequency $(f=50$ $\mathrm{Hz})$ and big velocity of strip movement $\left(v_{\mathrm{x}}=1 \mathrm{~m} / \mathrm{s}\right)$ velocity coefficient $k_{\mathrm{r}}$. reaches value of 3.12

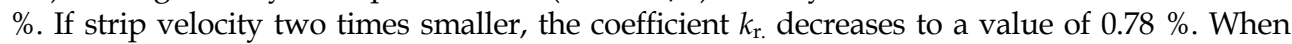
frequency of field current increases velocity coefficient $k_{\mathrm{r}}$ rapidly decreases. For frequency of field current $f=2000 \mathrm{~Hz}$ and velocity of strip movement $v_{\mathrm{x}}=1 \mathrm{~m} / \mathrm{s}$ velocity coefficient $k_{\mathrm{r}}=$ $0.0186 \%$ only. For the same frequency and two times smaler velocity $v_{\mathrm{x}}=0.5 \mathrm{~m} / \mathrm{s}$ velocity coefficient $k_{\mathrm{r}}=0.0047$. More detailed analysis of the subject was presented in (Barglik, 2005).

Based upon the presented above analysis equation (3) for magnetic vector potential could be transformed into the form (6)

$$
\operatorname{curl} \operatorname{curl} \underline{\boldsymbol{A}}+\mathrm{j} \omega \mu \gamma \underline{\boldsymbol{A}}=\underline{\boldsymbol{J}_{z}}
$$

Equation (6) should be completed by adequate boundary conditions for magnetic vector potential. For planes of external borders of the domain (BIJC, DCJK, I'KJI - see Fig.6) Dirichlet condition for magnetic vector potential should be satisfied:

$$
\underline{A}=0
$$

The same condition was applied for symmetry plane (I'ABI - see Fig.6) characterized by antisymmetry of field currents. For other symmetry planes of the calculation domain: $z=0$, ADKI' and $y=0, \mathrm{ABCD}$ (see Fig.6) condition (8) was applied:

$$
\underline{A} \times \boldsymbol{n}=\mathbf{0}
$$


a)

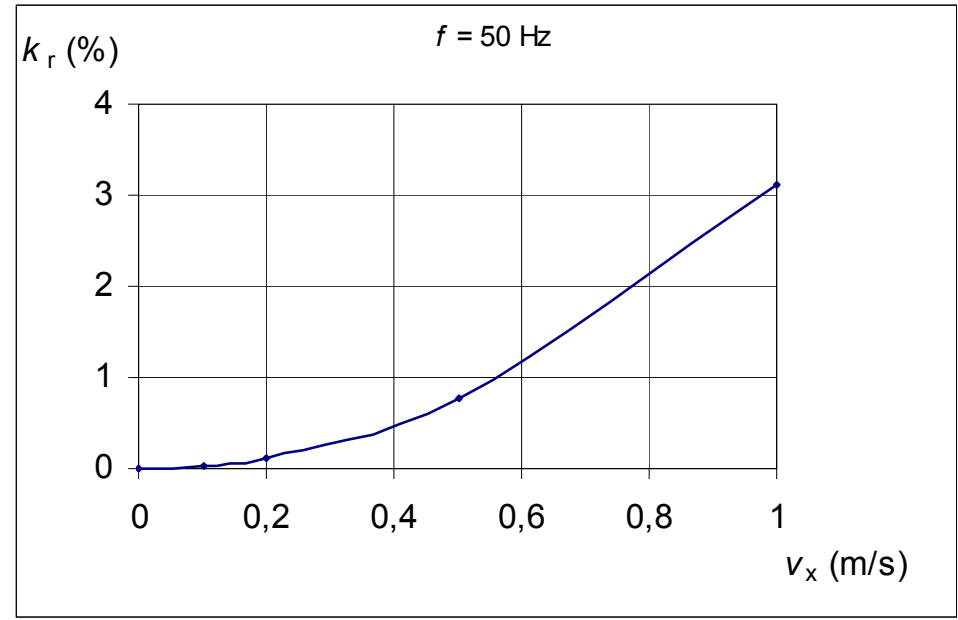

b)

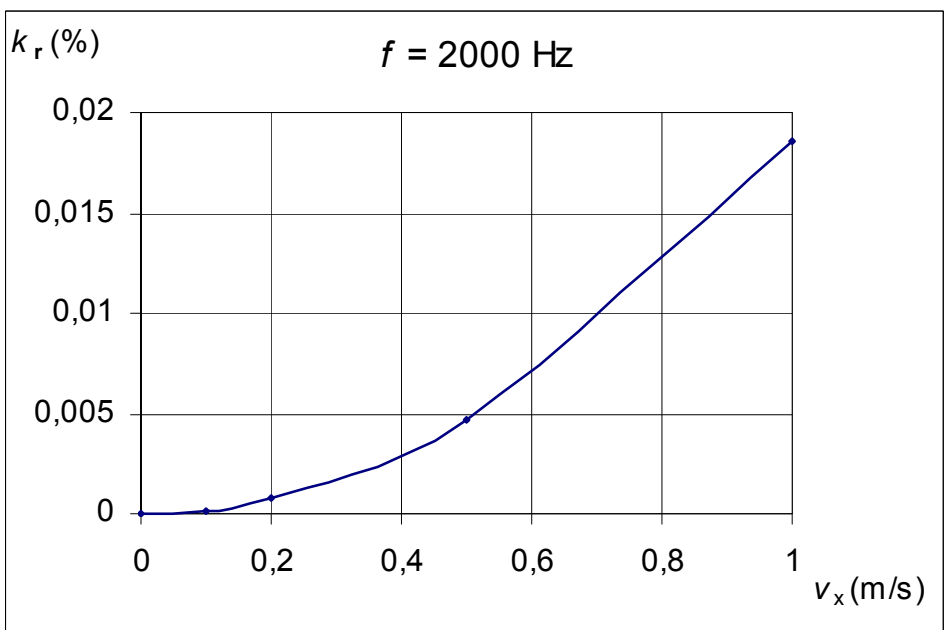

Fig. 7. Dependence of velocity coefficient on velocity of strip movement frequency of field current a) $f=50 \mathrm{~Hz}, \mathrm{~b}) f=2 \mathrm{kHz}$

Eddy current density induced in the strip $\underline{\boldsymbol{J}}$ and specific Joule losses $p_{\mathrm{v}}$ were expressed by equations (9-10)

$$
\begin{aligned}
& \underline{\boldsymbol{J}}=\mathrm{j} \omega \gamma \underline{\boldsymbol{A}} \\
& p_{\mathrm{v}}=\frac{\boldsymbol{J} \cdot \underline{\boldsymbol{J}^{*}}}{\gamma}
\end{aligned}
$$

where $\underline{\boldsymbol{J}}^{*}$ denotes the complex conjugate to $\underline{\boldsymbol{J}}$. 
Model for temperature field calculations was presented in Fig.8

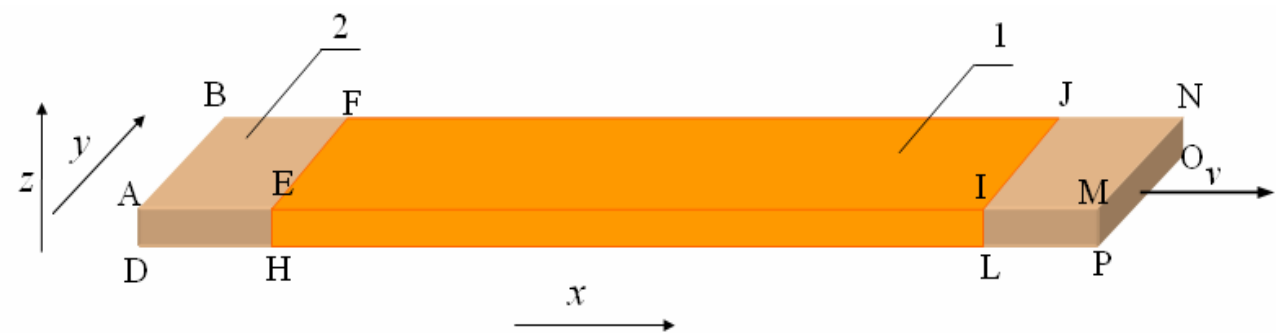

Fig. 8. Model for calculation of temperature field in the workpiece: 1 - part of the strip located inside air-gap of the inductor 2 - remaining part of the strip

As long as possible part of the moving strip was taken in order to correctly formulate boundary conditions on planes ABCD and MNOP (see Fig.8). Calculations were provided for $1 / 4$ part of the strip taking into account symmetry of temperature field to planes AMPD and DCOP (see Fig.8). Temperature distribution within the strip was determined by means of Kirchhoff-Fourier equation completed by specific Joule losses taken from electromagnetic calculations (Holman, 2009):

$$
\operatorname{div}(\lambda \operatorname{grad} T)-\rho c(v \operatorname{grad} T)=-p_{\mathrm{v}}
$$

where $\lambda$ denotes specific thermal conductivity, $\rho$ - density and $c$ - specific heat

Differential equation (11) was completed by a set of boundary conditions. At the artificial boundary plane ABCD (see Fig. 8) located sufficiently far from the inductor the condition (12) should be satisfied:

$$
T(y, z, t)=T_{\mathrm{p}}
$$

where $T_{\mathrm{p}}$ denotes temperature of strip before heating.

At the second artificial boundary plane MNOP (see Fig.8) located also sufficiently far from the inductor the condition (13) should be satisfied:

$$
\frac{\partial T}{\partial x}=0
$$

At part 1 of upper plane of the strip located in the narrow air-gap of the inductor FJIE (see Fig. 8) classical boundary condition for convection was satisfied. Radiation was neglected

$$
-\lambda \frac{\partial T}{\partial y}=\alpha_{\mathrm{g}}\left(T-T_{\mathrm{o}}\right)
$$

where $\alpha_{\mathrm{g}}$ denotes convection heat transfer coefficient in a narrow air-gap, $T_{\mathrm{o}}-$ ambient temperature.

Coefficient $\alpha_{\mathrm{g}}$ in a narrow air-gap of the inductor was several times smaller than outside the inductor (Barglik, 2005). Neglecting of radiation seems to be reasonable hovewer it could a source of additional errors (Barglik et al, 2008). So for other external planes of the strip 
ABFE, IJNM, BNOC (see Fig. 8) boundary conditions were formulated in a simplified way both for convection and radiation by introduction of total heat transfer coefficient $\alpha_{\mathrm{z}}$

$$
-\lambda \frac{\partial T}{\partial n}=\alpha_{\mathrm{z}}\left(T-T_{\mathrm{o}}^{\prime}\right)
$$

where $T_{\mathrm{o}}^{\prime}$ denotes average ambient temperature representing both radiation and convection. Total coefficient $\alpha_{\mathrm{z}}$ was given by relation (16)

$$
\alpha_{\mathrm{z}}=\alpha_{\mathrm{k}}+\sigma_{\mathrm{o}} \cdot\left(T^{2}+T_{\mathrm{r}}^{2}\right) \cdot\left(T+T_{\mathrm{r}}\right)
$$

where $\alpha_{\mathrm{k}}$ denotes convection heat transfer coefficient, $\sigma_{\mathrm{o}}$ - Stefan-Boltzman constant, $T_{\mathrm{r}}-$ temperature of surrounding surfaces involved in radiation heat transfer phenomena.

At both symmetry planes (AMPD, DCOP - see Fig.8) condition (17) should be satisfied:

$$
\frac{\partial T}{\partial n}=0
$$

In order to calculate electrical efficiency of the inductor-workpiece system was enough to take into consideration simplified two-dimensional calculation model shown in Fig.9. Phenomena in width of the strip could be neglected.

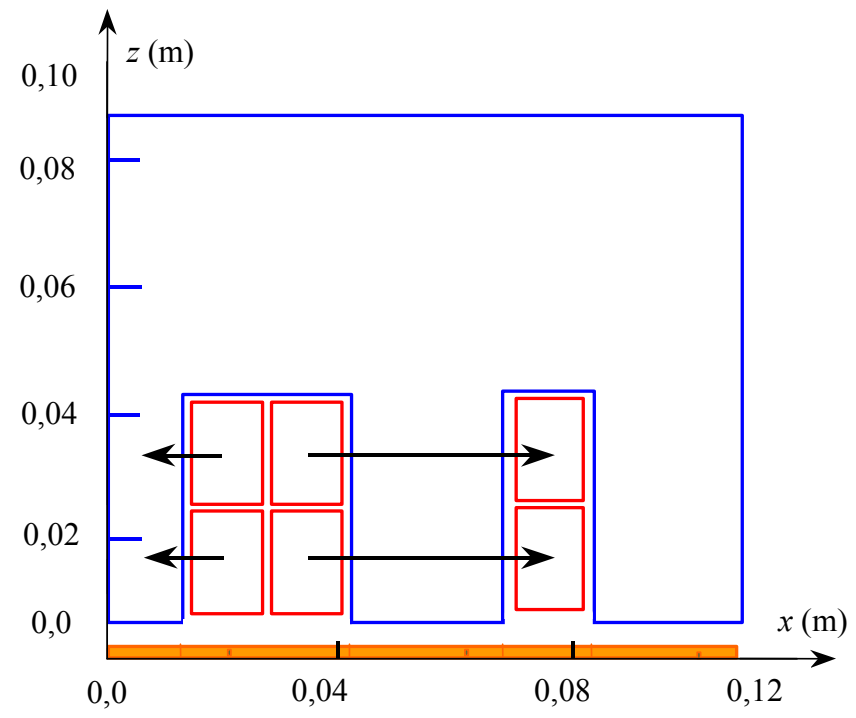

Fig. 9. Model for determination of electrical efficiency of inductor-workpiece system

Electrical efficiency of the inductor-workpiece system $\eta_{\mathrm{e}}$ were calculated by definition based upon equation (18)

$$
\eta_{\mathrm{e}}=\frac{\int_{V_{\mathrm{s}}} p_{\mathrm{v}} \mathrm{d} V_{s}}{\operatorname{Re}\{\boldsymbol{E} \times \boldsymbol{H}\} \mathrm{d} V_{\mathrm{t}}}
$$


where $V_{\mathrm{s}}$ - volume of the strip, Re - real part of phasor, $E$ - vector of electric field intensity, $\boldsymbol{H}$ - vector of magnetic field intensity, $V_{\mathrm{t}}$ - total volume of the system

\section{Illustrative example}

Based upon the presented above model:

- temperature distribution within the moving strip,

- electrical efficiency of the inductor-workpiece system

were calculated. For the temperature calculations 3D model was used in weak-coupled formulation. First, the distribution of electromagnetic field was calculated by means of FEMbased software OPERA 3D. Eddy current, specific Joule losses and finally temperature distribution were determined. Non-linear dependencies on temperature of material properties and parameters characterized heat transfer were taken into account. For calculations of electrical efficiency of the transverse flux induction heating system 2D model in quasi-coupled formulation was used. Calculations were done for many variants. Only small part of them was presented in the paper. Some basic input parameters and main dimensions of the system were presented below:

- frequency of field current $f=(50-3000 \mathrm{~Hz})$,

- $\quad$ inductor current $I=1000 \mathrm{~A}$,

- length of inductor $l_{\mathrm{i}}=0.217 \mathrm{~m}$,

- $\quad$ total width of inductor $b_{\mathrm{i}}=0.35 \mathrm{~m}$,

- $\quad$ width of magnetic core of the inductor $b_{c}=0.25 \mathrm{~m}$,

- height of inductor (half of two-side inductor) $h_{i}=0.08 \mathrm{~m}$,

- relative magnetic permeability of the magnetic core $\mu_{\mathrm{r}}=1000$,

- thickness of the air-gap of the inductor $a=0.01 \mathrm{~m}$ or $a=0.02 \mathrm{~m}$,

- material: copper, brass (Cu60), aluminium,

- length of the strip taken to the calculations $l=0.34 \mathrm{~m}$,

- strip thickness $g=0.0032 \mathrm{~m}$ or $g=0.002 \mathrm{~m}$ or $g=0.001 \mathrm{~m}$ or $g=0.0005 \mathrm{~m}$,

- $\quad$ strip velocity $v=0.01$ or $v=0.02$ or $v=0.04 \mathrm{~m} / \mathrm{s}$,

- ambient temperature $T_{0}=20^{\circ} \mathrm{C}$,

- temperature on inlet of the inductor $T_{\mathrm{p}}=20^{\circ} \mathrm{C}$,

- material properties in ambient temperature,

- $\quad$ electric conductivity: copper $\gamma_{\mathrm{Cu}}=5.7 \cdot 10^{7} \mathrm{~S} / \mathrm{m}$, brass $\gamma_{\mathrm{b}}=1.43 \cdot 10^{7} \mathrm{~S} / \mathrm{m}$, aluminum $\gamma_{\mathrm{Al}}=3.7 \cdot 10^{7} \mathrm{~S} / \mathrm{m}$,

- $\quad$ relative magnetic permeability $\mu_{\mathrm{r}}=1$,

- density: copper $\rho_{\mathrm{Cu}}=8900 \mathrm{~kg} / \mathrm{m}^{3}$, brass $\rho_{\mathrm{b}}=8600 \mathrm{~kg} / \mathrm{m}^{3}$, aluminum $\rho_{\mathrm{Al}}=2700 \mathrm{~kg} / \mathrm{m}^{3}$,

- thermal conductivity: copper $\lambda_{\mathrm{Cu}}=395 \mathrm{~W} / \mathrm{mK}$, brass $\lambda_{\mathrm{b}}=144 \mathrm{~W} / \mathrm{mK}$, aluminum $\lambda_{\mathrm{Al}}=211 \mathrm{~W} / \mathrm{mK}$,

- specific heat: copper $c_{\mathrm{Cu}}=381 \mathrm{~J} / \mathrm{kgK}$, brass $c_{\mathrm{b}}=410 \mathrm{~J} / \mathrm{kgK}$, aluminum $c_{\mathrm{Al}}$ $933 \mathrm{~J} / \mathrm{kgK}$,

- convection heat transfer coefficient in a narrow gap $a_{g}=5 \mathrm{~W} / \mathrm{m}^{2} \mathrm{~K}$

- convection heat transfer coefficient outside the inductor $a_{k}=20 \mathrm{~W} / \mathrm{m}^{2} \mathrm{~K}$,

- Stefan -Boltzmann constant $\sigma_{\mathrm{o}}=5.6676 \cdot 10^{-8} \mathrm{~W} /\left(\mathrm{m}^{2} \cdot \mathrm{K}^{4}\right)$

As it was mentioned earlier non-linear characteristics on temperature of all material properties were taken into account. 


\subsection{Mains frequency induction heating}

Distribution of eddy-current density for two different widths of the brass strip was shown in Fig. 10.
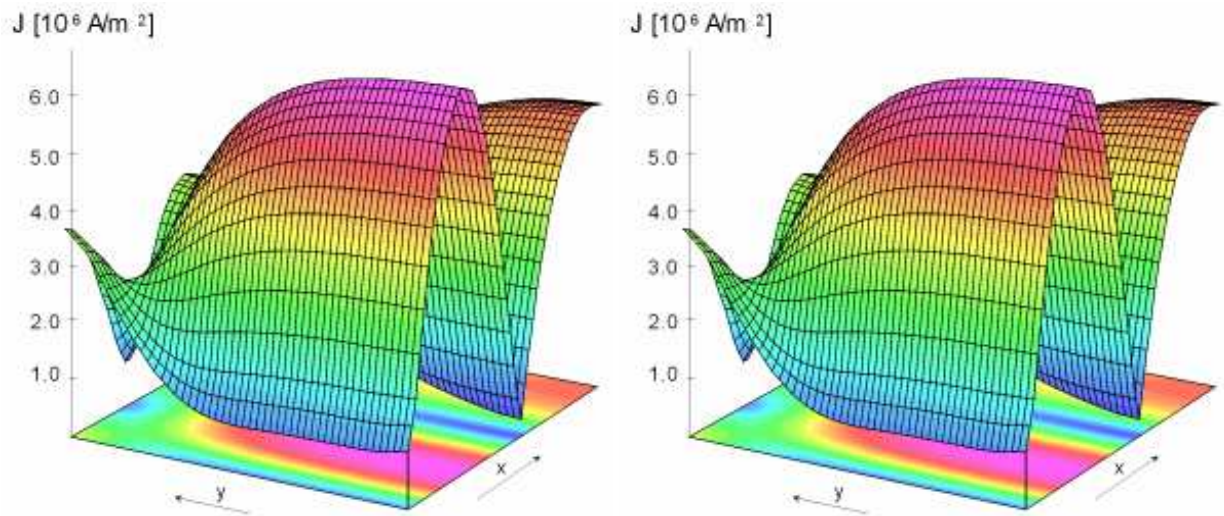

Fig. 10. Distribution of absolute value of eddy current density at internal plane of flat brass workpiece for two different widths of the brass strip $b=0.35 \mathrm{~m}$ (left histogram), $b=0.25 \mathrm{~m}$ (right histogram).

In the first case shown in left histogram of Fig. 10 the width of the strip was equal to the total width of the inductor. In the second case shown in right histogram of Fig. 10 the width of the strip is equal to the width of the magnetic core. Absolute value of eddy current density reaches its maximum exactly in an axis of the workpiece. In both cases the distribution of eddy current was strongly non-uniform. Let us calculate a coefficient of nonuniformity of eddy current distribution defined as an absolute value of eddy currents in the axis of the strip to absolute value of eddy currents near its edges:

$$
k_{\mathrm{J}}=\left.\frac{J_{y=0}}{J_{\mathrm{y}=\mathrm{b}}}\right|_{x=x_{\max }}
$$

where $x_{\max }$ denotes such a value of coordinate $x$ for which abslolute value of eddy current density reaches its maximum. Results were selected in Tab. 1

Based upon comparison of two cases characterized by different width of the strip it was assumed that the distribution of eddy current density was more uniform in case of narrow strip. Distribution of specific Joule losses released in the brass strip was presented in Fig. 11. Parameters describing specific Joule losses distribution were collected in Tab. 2. Coefficient of non-uniformity of specific Joule losses $k_{\mathrm{p}}$ was defined as a ratio of value of specific Joule losses in an axis if the strip to the value of specific Joule losses near the edge of the strip.

$$
k_{\mathrm{p}}=\left.\frac{p_{\mathrm{v}_{y=0}}}{p_{\mathrm{v}_{\mathrm{y}=\mathrm{b}}}}\right|_{x=x_{\max }}
$$

In both presented cases average values of specific Joule losses seem to be practically the same. But non-uniformity of distribution of specific Joule losses was relatively bigger. 


\begin{tabular}{|c|c|c|c|}
\hline \multirow{2}{*}{$\begin{array}{c}\text { Absolute value of } \\
\text { eddy current } \\
\begin{array}{c}\text { density } \\
\left(10^{6} \mathrm{~A} / \mathrm{m}^{2}\right)\end{array}\end{array}$} & Value & \multicolumn{2}{|c|}{ Width $b(\mathrm{~m})$} \\
\cline { 2 - 4 } & $\begin{array}{c}\text { average } J_{\mathrm{a}} \\
\text { for } x_{\max }=0.0275 \mathrm{~m}\end{array}$ & 0.35 & 0.25 \\
\cline { 2 - 4 } & $\begin{array}{c}\text { near the edge } J_{\mathrm{y}=\mathrm{b}} \\
\text { for } x_{\max }=0.0275 \mathrm{~m}\end{array}$ & 1.96 & 3.14 \\
\hline \multicolumn{2}{|c|}{ Coefficent of non-uniformity $k_{\mathrm{J}}(-)$} & 3.63 & 1.819 \\
\hline
\end{tabular}

Table 1. Parameters describing non-uniformity of eddy current distribution $(f=50 \mathrm{~Hz})$

\begin{tabular}{|c|c|c|c|}
\hline \multirow{4}{*}{$\begin{array}{c}* \\
\text { specific Joule } \\
\text { losses } \\
\left(10^{7} \mathrm{~W} / \mathrm{m}^{3}\right)\end{array}$} & Value & \multicolumn{2}{|c|}{ width $b(\mathrm{~m})$} \\
\cline { 2 - 4 } & average $p_{\mathrm{vss}}$ & 0.35 & 0.25 \\
\cline { 2 - 4 } & $\begin{array}{c}\text { in the axis } p_{\mathrm{v}(\mathrm{y}=0)} \\
\text { for } x_{\max }=0.0275 \mathrm{~m}\end{array}$ & 4.067 & 1.264 \\
\cline { 2 - 4 } & $\begin{array}{c}\text { Near the edge } p_{\mathrm{v}(\mathrm{y}=\mathrm{b})} \\
\text { For } x_{\max }=0,0275 \mathrm{~m}\end{array}$ & 0.32 & 2.096 \\
\hline \multicolumn{2}{|c|}{ Non-uniformity coefficient $k_{\mathrm{p}}(-)$} & 13.1 & 2.03 \\
\hline
\end{tabular}

Table 2. Parameters describing non-uniformity of specific Joule losses distribution

For the first case of induction heating of the broader strip $(b=0.35 \mathrm{~m})$ coefficient of nonuniformity of specific Joule losses $k_{\mathrm{p}}=13.1$, however fo the second case of transverse flux induction heating of the narrower strip the coefficient seems to be several times smaller $\left(k_{\mathrm{p}}=2.03\right)$.

Some examplary results of specific Joule losses distribution in the moving brass strip were shown in Fig. 11. Non-uniform specific Joule losses distribution in the strip width was observed. In both cases the specific Joule losses reaches its maximum in the axis of the strip. Shape of the curie strongly depends on the width of the strip. More precisely speaking it
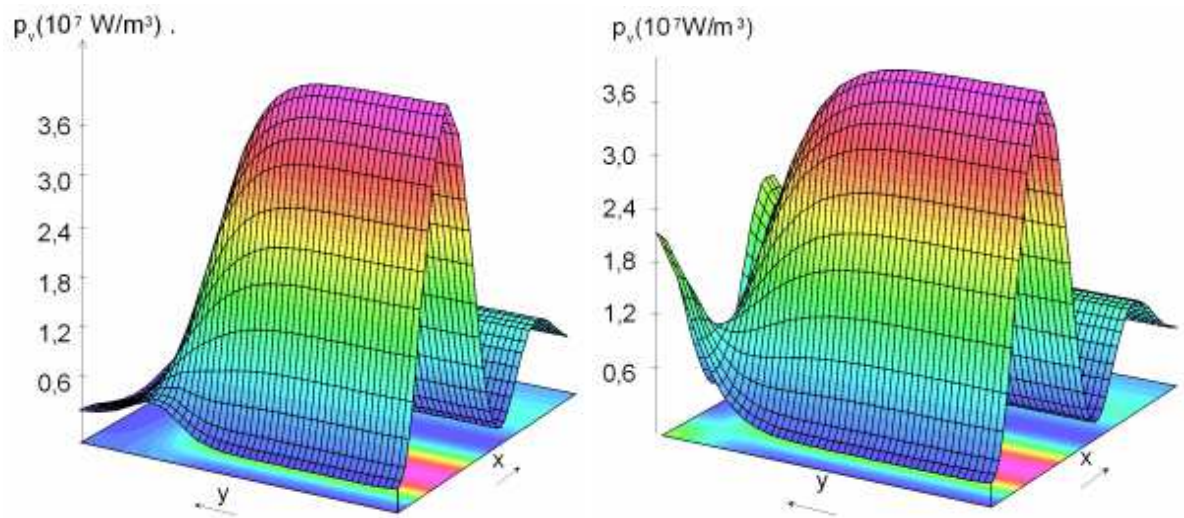

Fig. 11. Specific Joule losses for two different strip widths $b=0.35 \mathrm{~m}$ (left histogram), $b=0.25 \mathrm{~m}$ (right histogram) 

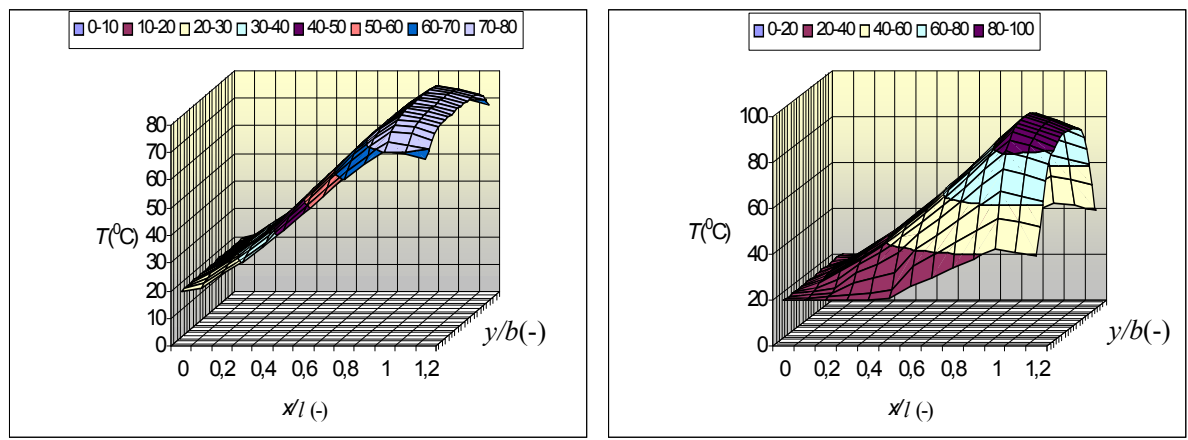

Fig. 12. Temperature distribution on upper plane of brass strip heated by induction moving with velocity $v=0.01 \mathrm{~m} / \mathrm{s}$ for two different widths $b=0.35 \mathrm{~m}$ (left diagram) and $b=0.25 \mathrm{~m}$ (right diagram)

depends on the ratio between the width of the strip and the total width of the inductor. In case of broader strip $\left(b=b_{\mathrm{i}}\right)$ decrease of temperature seems to be more clear (about $53 \%$ at the outlet of the inductor). In case of narrower strip decrease of temperature was distincly smaller $\left(b=b_{c}\right)$ reaching of about $11 \%$. Applied supply system with mains frequency makes it possible to heat up the strip to relatively low temperature (see Fig.12), even for relatively small velocity of strip movement. In order to obtain big temperatures it is necessary to increase the frequency of field current.

\subsection{Medium frequency transverse flux induction heating}

The most important parameter strongly influenced on uniformity of eddy current distribution and consequently on specific Joule losses was the frequency of field current. Distribution of specific Joule losses at the internal plane of brass strip for two selected frequencies of field current $(f=200 \mathrm{~Hz}$ and $f=2000 \mathrm{~Hz})$ was shown in Fig. 13 .
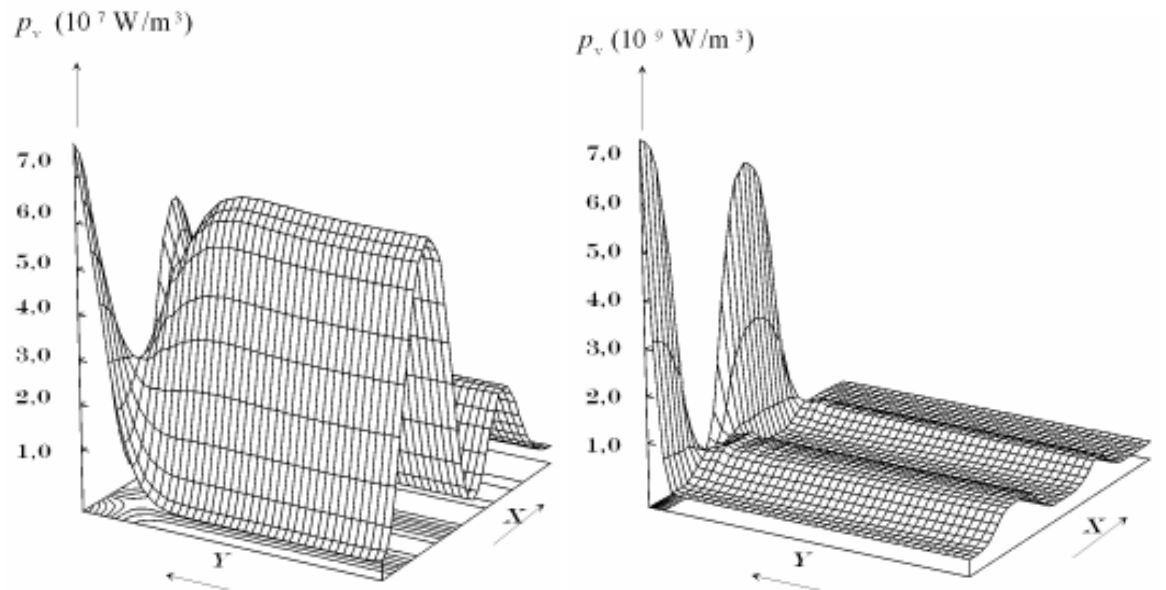

Fig. 13. Distribution of specific Joule losses at internal plane of brass strip $(b=0.25 \mathrm{~m})$ for $f=200 \mathrm{~Hz}$ (left histogram) and $f=2000 \mathrm{~Hz}$ (right histogram) 
Parameters describing distribution of specific Joule losses in the workpiece for three different frequencies were presented in tab. 3

\begin{tabular}{|c|c|c|c|c|c|c|}
\hline \multirow{3}{*}{ Specific Joule losses $\left(10^{7} \mathrm{~W} / \mathrm{m}^{3}\right)$} & \multicolumn{6}{|c|}{ Frequency $f(\mathrm{~Hz})$} \\
\hline & \multicolumn{3}{|c|}{ Brass } & \multicolumn{3}{|c|}{ Aluminum } \\
\hline & 50 & 200 & 2000 & 50 & 200 & 2000 \\
\hline Average value $p_{\mathrm{v}, \mathrm{av}}$ & 1.264 & 5.4 & 15.12 & 2.11 & 6.4 & 15.82 \\
\hline $\begin{array}{l}\text { Value at the axis }\left.p_{\mathrm{v}}\right|_{y=0} \\
\text { for } x_{\max }=0.0275 \mathrm{~m}\end{array}$ & 4.096 & 6.3 & 10.18 & 3.1 & 6.8 & 10.5 \\
\hline $\begin{array}{l}\text { Value at the axis }\left.p_{\mathrm{v}}\right|_{y=b} \\
\text { for } x_{\max }=0.0275 \mathrm{~m}\end{array}$ & 2.02 & 7.05 & 72.45 & 2.5 & 9.5 & 91.45 \\
\hline $\begin{array}{l}\text { Coefficient of non-uniformity } k_{\mathrm{p}} \\
(-)\end{array}$ & 2.03 & 0.888 & 0.14 & 1.24 & 0.674 & 0.1148 \\
\hline
\end{tabular}

Table 3. Parameters describing specific Joule losses in the workpiece

If frequency of field current increases average value of specific Joule losses also increase. If we try to compare shapes of specific Joule losses histograms for different field current frequencies we learn about big differences between them. For mains frequency $(f=50 \mathrm{~Hz})$ (see Fig. 11 right histogram) specific Joule losses reach their maximum at the axis of the strip. For bigger frequency $f=200 \mathrm{~Hz}$ distribution of specific Joule losses (see Fig. 13 left histogram) seems to be more uniform. Difference between maximum value reached near edge of the strip and value at the axis is rather small. Coefficient of non-uniformity is equal to 0.888. At bigger frequency (Fig. 13 - right drawing) distribution of the specific Joule losses become again strongly non-uniform with a distinct maximum near the edge of the strip. Coefficient of non-uniformity was equal to 0.138 for that case. Exemplary results of temperature calculations in moving strip for frequency of field current $f=2000 \mathrm{~Hz}$ was shown in Fig. 14.
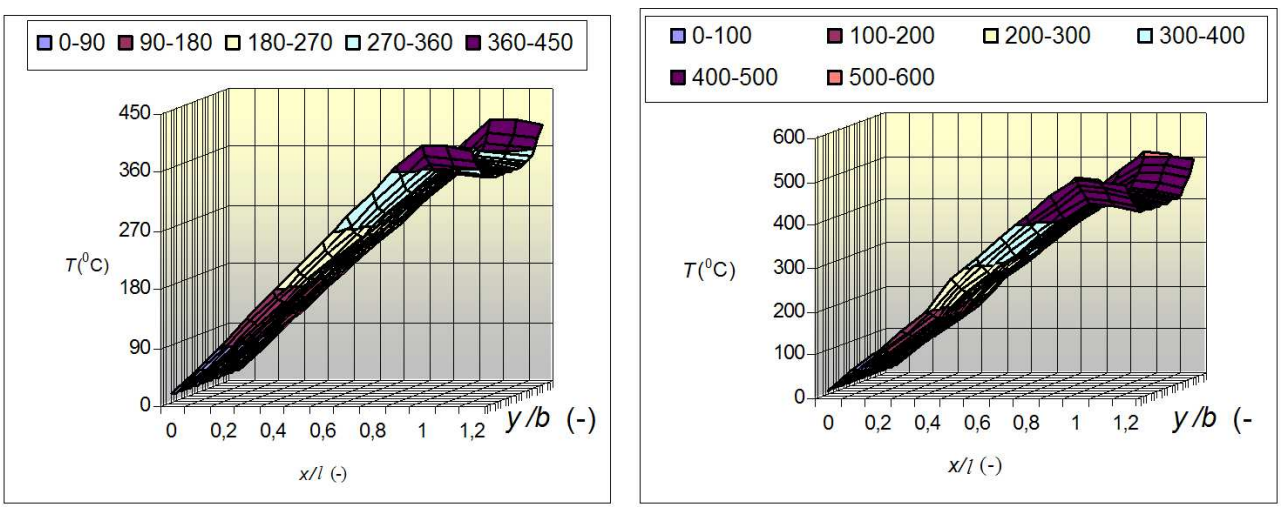

Fig. 14. Temperature distribution at upper plane of brass (left diagram) and aluminum (right diagram) strip

Non-uniform temperature distribution in width of the strip was observed with maximum near the edges. In we take into consideration the criterion of temperature distribution 
uniformity we could assume that it was only one frequency of field current being the optimal one from that point of view. For presented cases of induction transverse flux heating the optimal frequency of field current was about $150-300 \mathrm{~Hz}$. For lower frequencies shape of temperature curve was again non-uniform with the maximum near the axis of the strip. The optimal frequency for transverse flux induction heating of brass strip of thickness $g=3.2 \mathrm{~mm}$ was $275 \mathrm{~Hz}$ (Barglik, 2002) an for aluminium strips of same thickness was slightly smaller $\left(f_{\text {opt }}=175 \mathrm{~Hz}\right)$ (Barglik, 2005).

\subsection{Electrical efficiency}

As it was mentioned earlier for calculation of electrical efficiency of the transverse flux inductor-workpiece system a simplified two-dimensional calculation model for used. Below were presented some results of the calculations. Dependence of electrical efficiency of the inductor-brass strip system on frequency for two different values of relative air-gaps of the inductor defined as a ratio of thickness of the inductor air-gap $a$ and the thickness of the strip $g$ was shown in Fig. 15.

$$
a^{\prime}=\frac{a}{g}
$$

For induction heating of the strip with thickness $g=5 \mathrm{~mm}$ electrical efficiency reaches its maximum for frequency of field current $f \approx 100 \mathrm{~Hz}$. In case of strip with thickness $g=3.2$ $\mathrm{mm}$ the maximal value of the electrical efficiency of the system was three times bigger $(f \approx 300 \mathrm{~Hz})$.

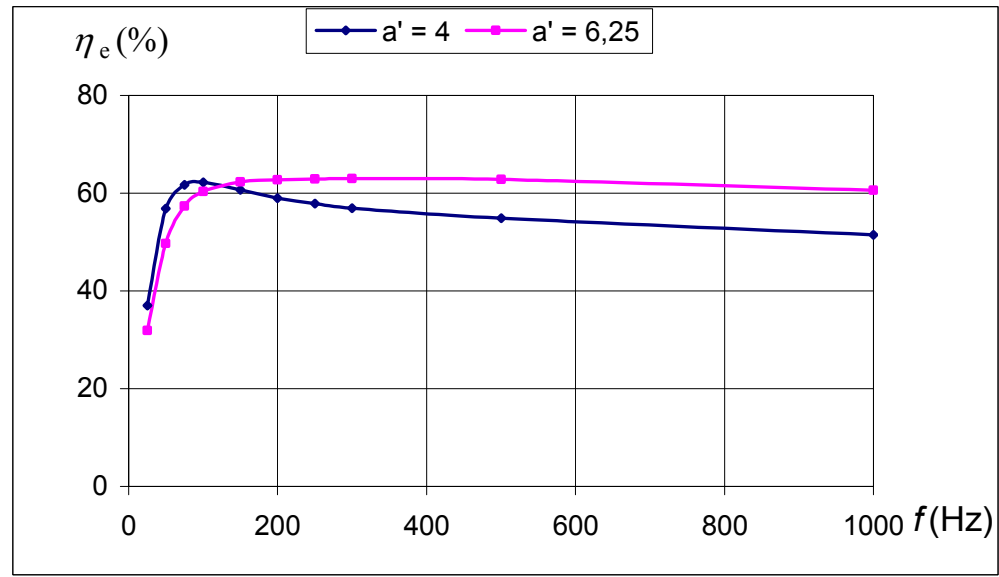

Fig. 15. Dependence of electrical efficiency of inductor-brass strip for two relative air-gap of the inductor-strip system ( $\mathrm{a}=20 \mathrm{~mm}, g=3.2 \mathrm{~mm}, 5 \mathrm{~mm})$.

Dependence of electrical efficiency of inductor-brass strip system for heating of strips with thicknesses: $g=1 \mathrm{~mm}, 2 \mathrm{~mm}$ was presented in Fig. 16 


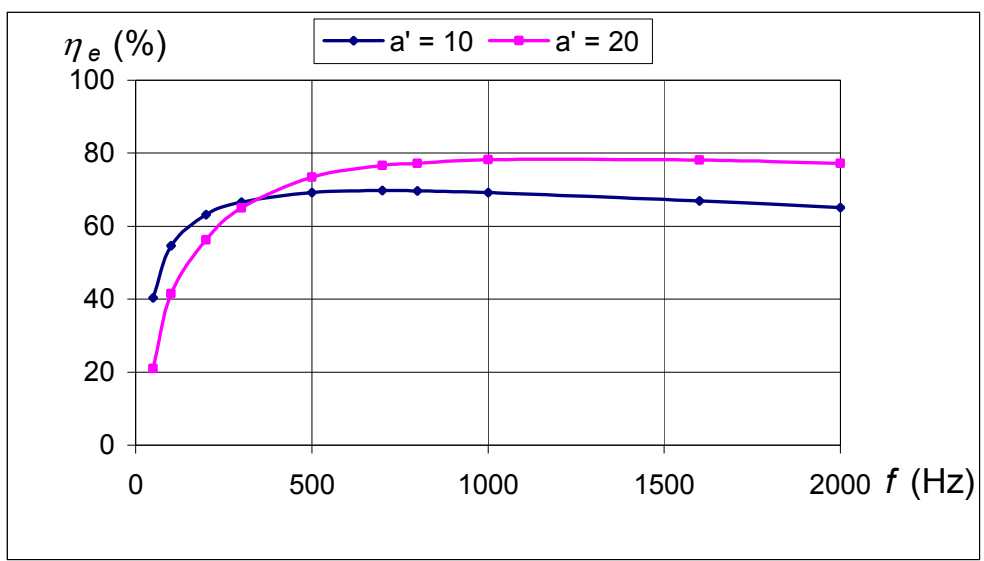

Fig. 16. Dependence of electrical efficiency of inductor-brass strip system on field current frequency for $g=2 \mathrm{~mm}, a^{\prime}=10$ and $g=1 \mathrm{~mm}, a^{\prime}=20$

The air-gap of the transverse flux inductor was again $a=20 \mathrm{~mm}$. Electrical efficiency of the transverse flux inductor-strip system $\eta_{\mathrm{e}}$ reaches its maximum at bigger frequencies of field current. For thickness of the strip $g=2 \mathrm{~mm}$ and the relative air-gap of the inductor $a^{\prime}=10$, the electrical efficiency of the system reaches its maximum $\left(\eta_{\mathrm{e}}=\eta_{\max }\right)$ at frequency of field current $f \approx 700 \mathrm{~Hz}$. For smaller strip thickness $g=1 \mathrm{~mm}$ and the relative air-gap of the inductor $\mathrm{a}^{\prime}=20$ the electrical efficiency of the system reaches its maximal value $\left(\eta_{\mathrm{e}}=\eta_{\max }\right)$ for almost two times bigger frequency of field current $f \approx 1300 \mathrm{~Hz}$.

Dependence of electrical efficiency of the transverse flux inductor-brass strip system for two kinds of the heated material: brass and copper was presented in Fig. 17. Comparison was provided for very thin strips (thickness of the strip $g=0.5 \mathrm{~mm}$ ) heated in the basic configuration of the inductor (air-gap of the inductor $a=20 \mathrm{~mm}$ ). So the relative air-gap of the inductor strip-system was big $\left(a^{\prime}=40\right)$.

During the transverse flux induction heating of very thin brass strips maximum of electrical efficiency of the system $\eta_{\mathrm{e}}$ was reached at the frequency of field current $f \approx 2000 \mathrm{~Hz}$. For bigger frequencies of the field current the electrical efficiency of the transverse flux inductor - brass strip system slowly decreses. Similarly for smaller frequencies the electrical efficiency of the transverse flux inductor - brass strip system quickly decreases reaching value of only $\eta_{\mathrm{e}}=0.25$ for frequency of field current $f=50 \mathrm{~Hz}$. Completely different situation was in case of the transverse flux induction heating of similar copper strips. Maximal value of the electrical efficiency of the transverse flux inductor - copper brass system $\left(\eta_{\mathrm{e}}=\eta_{\max }=\right.$ 0.79 ) was obtained for frequency of field current $f \approx 200 \mathrm{~Hz}$. Then, for bigger frquencies $\eta_{\mathrm{e}}$ the electrical efficiency of the system slowly decreases up to the value of $\eta_{\mathrm{e}}=0.71$ for the frequency of field current $f \approx 200 \mathrm{~Hz}$. For the mains frequency of field current $f=50 \mathrm{~Hz}$ the electrical efficiency of the system was equal to $\eta_{\mathrm{e}}=0.7$. 


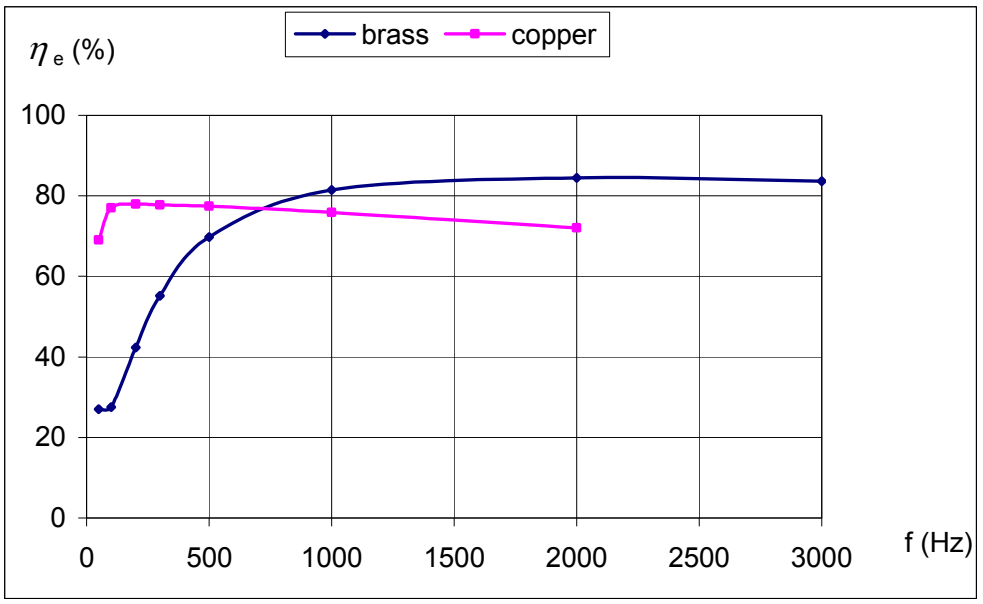

Fig. 17. Dependence of electrical efficiency of inductor - strip system for two kinds of material: brass and copper

Dependence of the electrical efficiency of the transverse flux inductor-strip system $\eta_{\mathrm{e}}$ on frequency of field current for brass strip (thickness $g=3.2 \mathrm{~mm}$ and air-gap of the inductor $a=10 \mathrm{~mm}$ ) was shown in Fig. 18

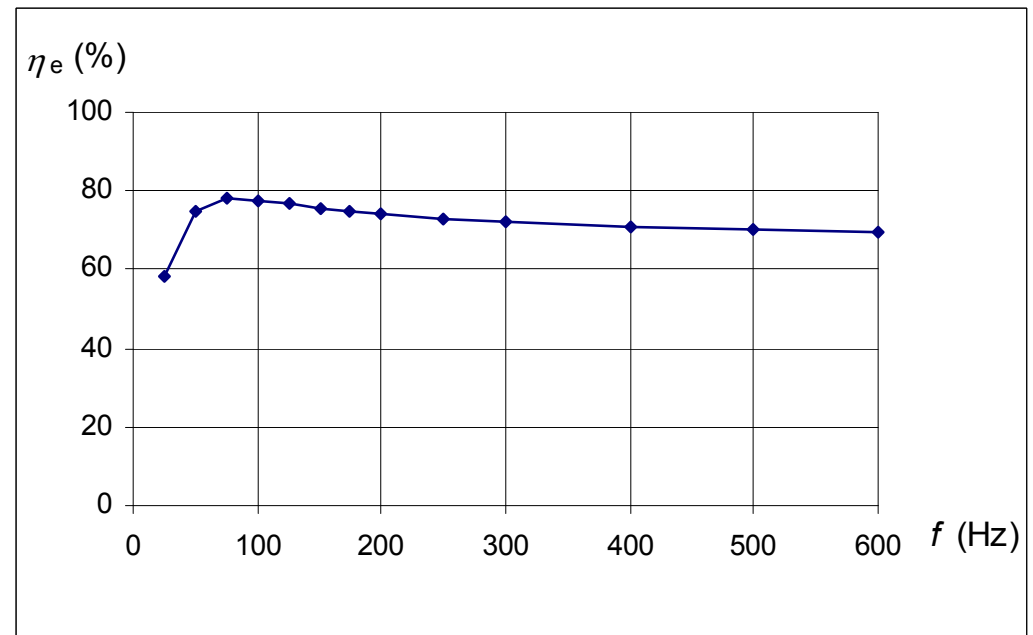

Fig. 18. Dependence of electrical efficiency of the inductor - brass strip system on field current frequency $(g=3.2 \mathrm{~mm}, a=10 \mathrm{~mm})$

For such a configuration of the system the maximum value of the electrical efficiency of the inductor-strip system was reached for frequency of field current of about $f \approx 75 \mathrm{~Hz}$. 


\section{Experimental part}

\subsection{Laboratory stand}

A block scheme of the laboratory stand equipped with a thyristor power source of medium frequency used for measurements necessary for experimental validation of computations was shown in Fig. 19. The similar laboratory stand not presented in the paper, making possible to supply the transverse flux induction heater by current of mains frequency was described in details in (Barglik, 2004). Brass strip (6) moves with the constant, but regulated velocity from an uncoiler drum (5) to a final drum (7) through the narrow air-gap of twoside, multipolar transverse flux inductor (4) (see Fig. 3 and Fig. 4). Velocity of strip movement was regulated fluently by means of stearing panel (9) containing two separate thyristor power sources: one of them for each direction of strip movement. In order to measure the final temperature of the strip $T_{\mathrm{k}}$ at the outlet of the inductor, pyrometer (8) was used. A specialized measured system (10) contaning converters making possible to register of measurement data in the proper database computer system (12) was applied. The thyristor power source (1) has its nominal active power of about $200 \mathrm{~kW}$ and frequency of harmonic field current regulated in a range of $(1-2.5 \mathrm{kHz})$ by means of block of capacitors (2). The transverse flux inductor was connected to the thyristor power source through the water cooled matching transformer (3).

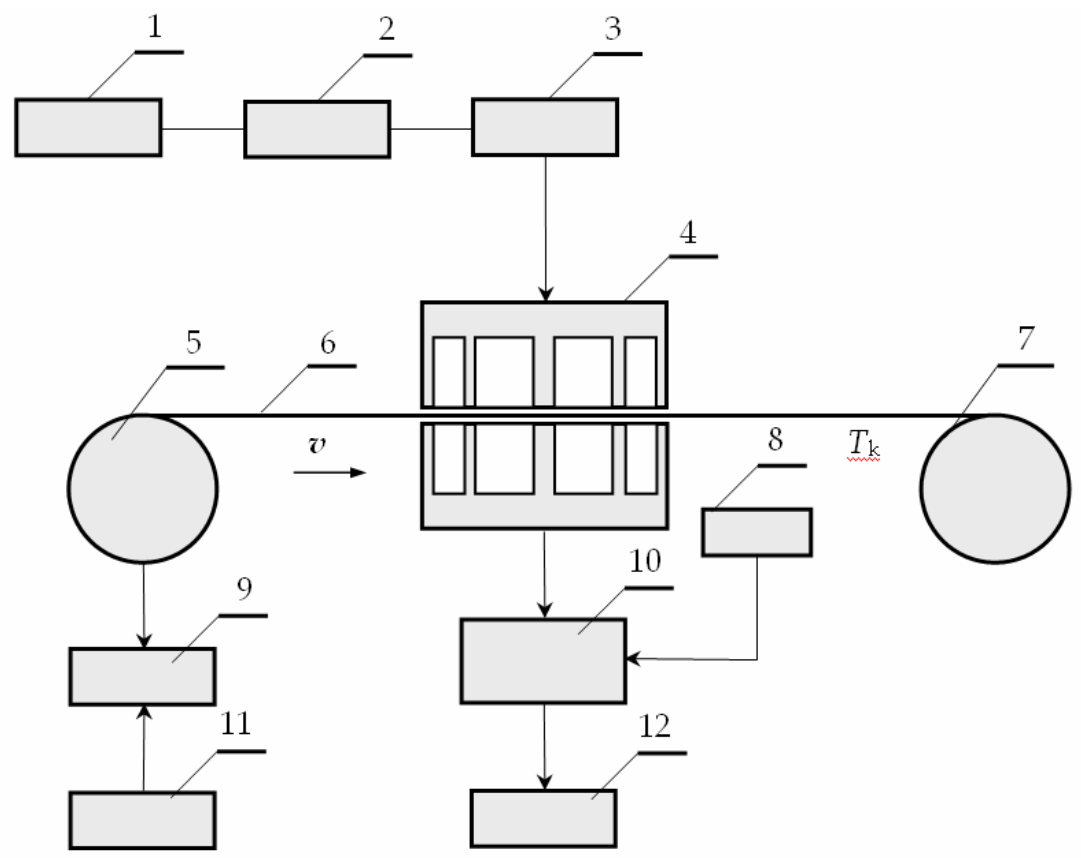

Fig. 19. Block scheme of the laboratory stand. 1- thyristor power source of medium frequency, 2 - block of capacitors, 3 - matching transformer 4 - transverse flux field inductor, 5 - uncoiler drum, 6 -brass strip, 7 - final drum, 8 -pyrometer, 9 - stearing panel, 10 - block of measurement converters, 11 - control desk, 12 - database computer system 
The overall view of the laboratory stand (block scheme in Fig. 19) was shown in Fig. 20. The stand was built in the laboratory of Electroheat of the Silesian University of Technology in Katowice, Poland. At the first plane of the photograph the control desk with the computer for registration of measurement have been seen. Then we could see the transverse flux inductor. It was placed at the special basis making possible a fluent regulation of the thickness of the inductor air-gap. At the last plane of the photograph we can see the thyristor power source and other elements of the supply system: the cubicle with capacitors (on left) and the matching transformer of medium frequency.

\subsection{Measurements}

The measurements were done at two separate laboratory stands. The transverse flux field inductor could be supplied in two ways: directly from $50 \mathrm{~Hz}$ regulated transformer (construction of such the laboratory stand was described in (Barglik, 2004)) or by field current of medium frequency through the thyristor power source, block of capacitors and the matching transformer (see Figs. 19, 20). The temperature distribution at the outlet of the inductor $(x=1.25 l)$ supplied by field current of mains frequency in the width of the moving brass strip for two different velocities of strip movement was shown in Fig. 21. Computations (blue line in Fig. 21) were compared with the measurements done at the laboratory stand (red points in Fig. 21). Characteristic was a shape of temperature curves

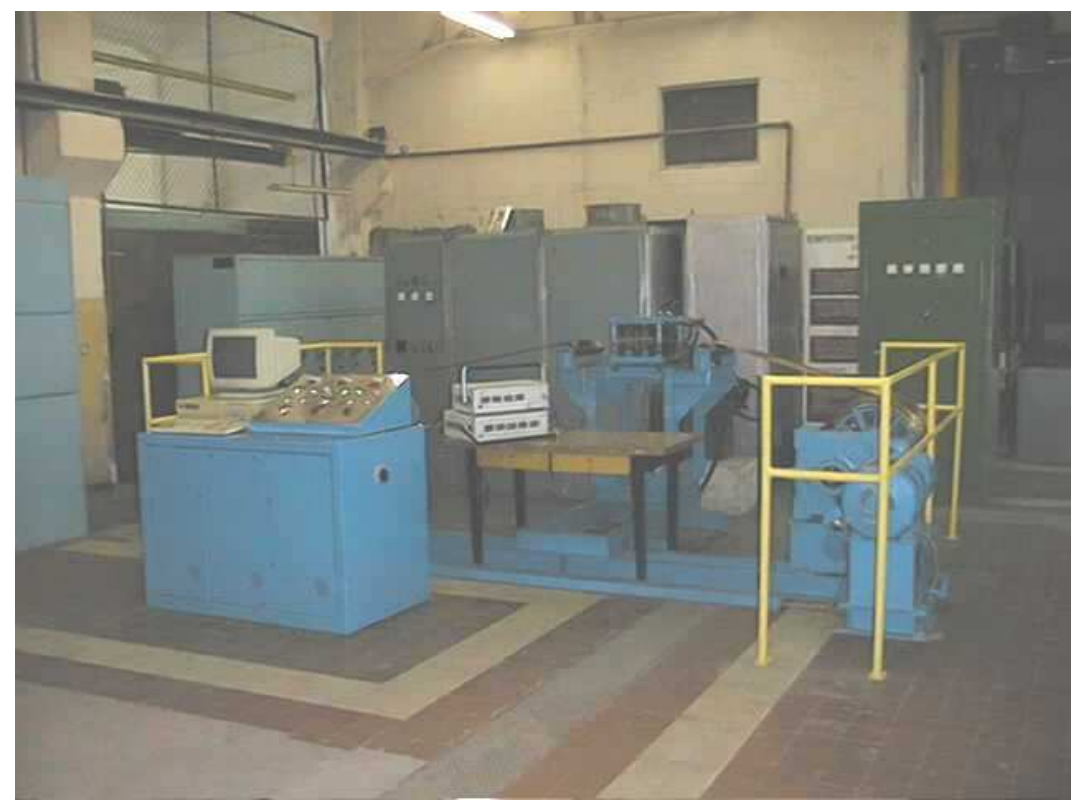

Fig. 20. View of the laboratory stand 

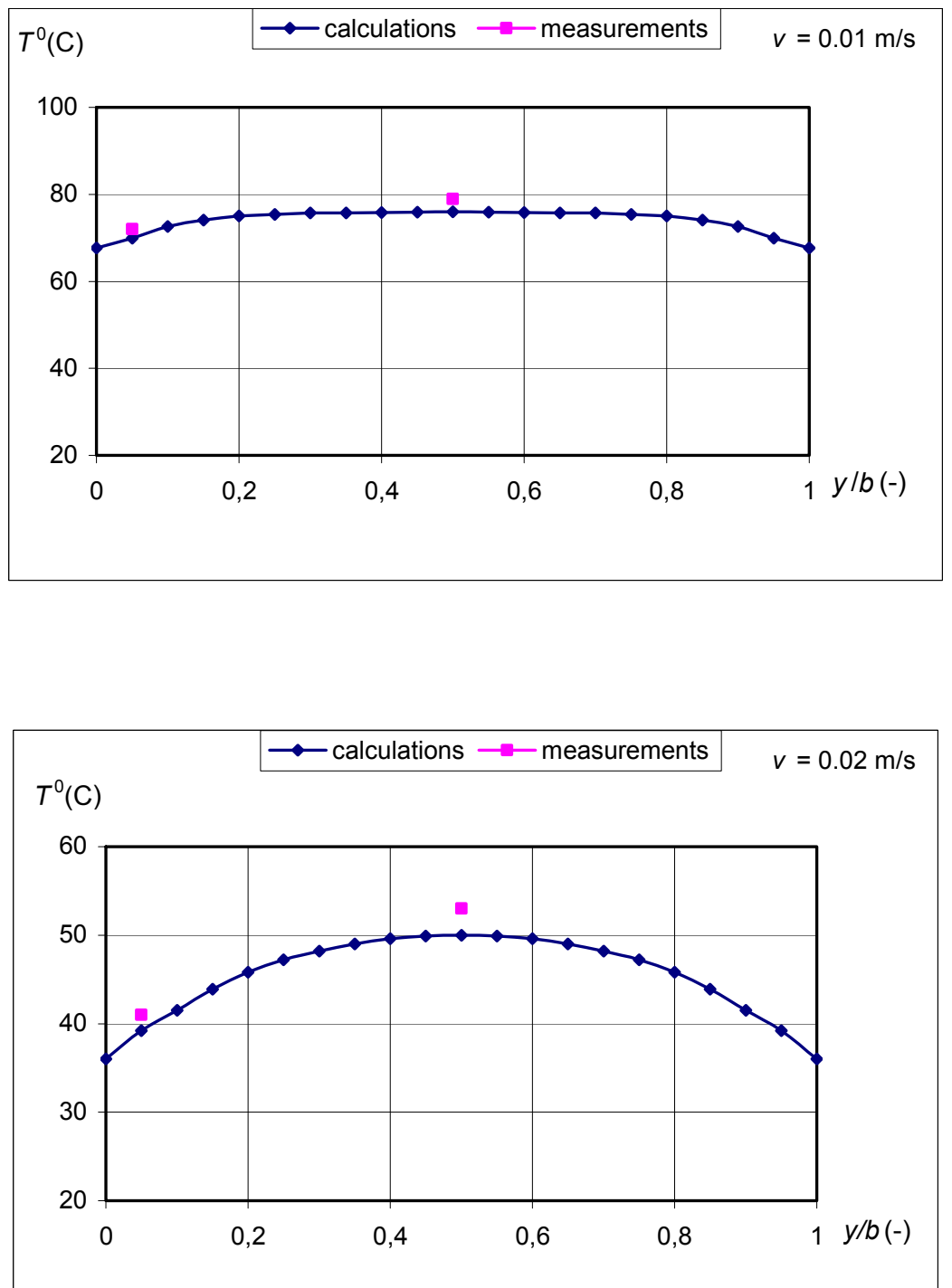

Fig. 21. Dependence of temperature on relative width of the brass strip at the outlet of the inductor $(x=1.25 \mathrm{l})$ supplied by current of mains frequency $(f=50 \mathrm{~Hz})$ for two different strip movement $(v=0,01 \mathrm{~m} / \mathrm{s}$ - upper diagram, $v=0,02 \mathrm{~m} / \mathrm{s}$ - lower diagram) 

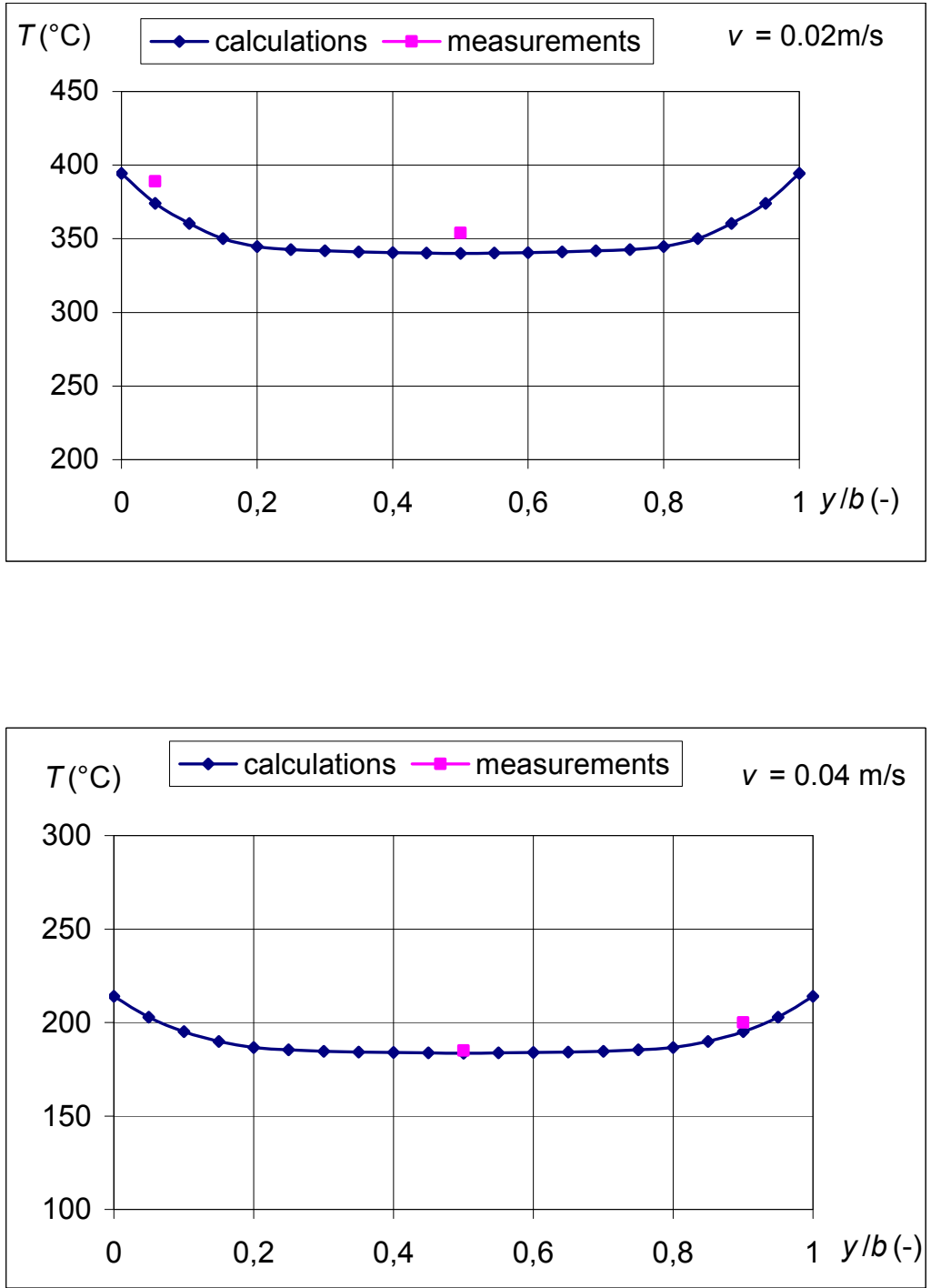

Fig. 22. Dependence of temperature on relative width of the bras strip. Frequency of field current $f=2000 \mathrm{~Hz}$. Velocity of strip movement $v=0,02 \mathrm{~m} / \mathrm{s}-$ upper diagram, $v=0,04 \mathrm{~m} / \mathrm{s}$ - lower diagram 
showing temperature distribution in width of the strip with the distinct maximum in the axis of the strip.

In both cases quite good accordance of about not more than $3.5 \%$ between calculations and measurements was achieved. The temperature distribution in width of the strip for medium frequency $(f=2000 \mathrm{~Hz})$ was shown in Fig. 22. Characteristic was a shape of temperature curves showing temperature distribution in width of the strip with the distinct maximum near the axis of the strip. In both presented cases a quite good accordance between calculations and measurements data of about $4.8 \%$ was achieved. In order to satisfy the criterion of uniform temperature distribution along the width of the strip it was necessary to find the optimal frequency of field current. As a result of computations such a frequency was obtained for a case of transverse flux induction heating of brass strip with thickness $g=3.2 \mathrm{~mm}$. It was equal to $f_{\mathrm{opt}}=275 \mathrm{~Hz}$ (Barglik, 2002). The uniform temperature distribution in width of the brass strip for optimal frequency of field current was obtained. The selection of optimal frequency based upon the criterion of temperature uniformity was valid for the particular configuration of the inductor-strip system. In case of usage of one inductor to heat up a wide range of the strips from materials of different properties and dimensions more complicated criteria should be applied (Barglik, 2002). Besides a classical division of the transverse flux inductor in a few single segments of different configuration to the axis of the strip it was also a concept of the flexible VABID inductor (Schülbe et al, 2004).

\section{Conclusions}

Three-dimensional analysis of electromagnetic and temperature fields in transverse flux induction heater for thin strips by means of FEM-based professional software supplemented by user codes was provided. Eddy current density and specific Joule losses released in the workpiece, temperature distribution in the width of the moving strip and the electrical efficiency of the transverse flux inductor-workpiece system were determined. The shapes of the temperature curves for different frequencies of field current were compared. The results were discussed in order to analyze possibilities of obtaining the requested parameters of the induction heating system. The computations were compared the measurement data. Quite good accordance between computations and measurements was noticed. Next steps in the investigations should be aimed at increasing the accuracy of calculations and shortening time of computations.

\section{Acknowledgement}

The financial support of the Polish Ministry of Science and Higher Education in several research projects realized in years 1995 - 2010 including ongoing projects N N510 256338 and N N508 388637 are highly grateful. Some research activities were done also within the framework of the multigovernment Polish-Czech collaboration project on mathematical modelling of induction heating processes.

\section{References}

Bakke, E. \& Nacke B. (2010). Efficient heating by electromagnetic sources in metallurgical processes; recent applications and development trends. Electrotechnic Review. No 7, July 2010, pp. 11-14 ISSN 0033-2097 
Baker, R. (1950). Transverse Flux Induction Heating, AIEE Transactions, vol. 69, pp. 711-719.

Barglik, J. (1992). Induction heating of thin non-ferrous strips, Proceedings of 37. Wissenschaftliche Kolloqium pp. 94-99, Ilmenau

Barglik, J. (2000) Influence of input material data on accuracy of thermal calculations at induction heating, Acta Technica CSAV, Vol. 45, pp. 323-336. ISSN 0001-7043

Barglik, J. (2002) Induction heating of flat charges in transverse flux field. Computer simulation and investigation verification. Scientific Issues of the Silesian University of Technology Vol.1575, No 65 (November 2002), pp. 1-120, PL ISSN 0324$802-\mathrm{X}$

Barglik, J. (2004). 3D analysis of coupled electromagnetic and temperature fields in transverse flux induction heating system supplied by current of mains frequency. Acta Technica, Prague, pp. 393-410, ISSN 0001-7043

Barglik, J. (2005). Computer modelling of induction heating of thin workpieces from nonferrous metals, In: Development in materials science and metallurgy. Publisher of the Silesian University of Technology. pp. 7-32 ISBN 83-7335-214-7. Gliwice

Barglik, J., Czerwiński, M., Hering, M. and Wesołowski,M. (2008). Radiation In Modelling of Induction Heating Systems. In: Advanced Computer Techniques in Applied Electromagnetics. IOS Press. pp. 202-211. ISSN 1383- 7281. Amsterdam, Berlin, Oxford, Tokyo, Washington, DC.

Barglik,J. (2010). Induction heating in technological processes - selected examples of applications Electrical Review, No 5 May 2010 pp.294 - 297 ISSN 0033-2097

Davies, E. (1990) Conduction and Induction Heating. Peter Peregrinus Ltd. on behalf of IEEE ISBN 0863411746 Exeter

Dughiero, F., Lupi S., Mühlbauer A. and Nikanorov A., (2003). TFH - Transverse Flux Induction Heating of Non-Ferrous and Precise Strips. Results of a EU research project,. Compel International Journal for Computation and Mathematics in Electrical Engineering., pp. 134 - 148 ISSN: 0332-1649

Hering, M. (1992). Basic of Electroheat. WNT 1998 ISBN 83-204-2319-8 Warsaw

Holman, J. (2009). Heat transfer, McGraw-Hill, ISBN 13: 9780073529363 New York

Jackson,W. (1972). Transverse Flux Induction Heating Flat Metal Products, Proceedings of the seventh UIE congress, Paper No 206. Warsaw, July 1972.

Mühlbauer. A, Leßmann H., Mohring, J., Demidowitch. V. Nikanorov (1995). Modelling of 3D Electromagnetic Processes in Transverse Flux Induction Heaters, Proceedings of Compumag Conference on Computation of Electromagnetic Fields, , pp. 444 - 445, Berlin

Mühlbauer, A., (2008). History of Induction Heating \& Melting, Vulkan-Verlag GmbH, ISBN 978-3-8027-2946-1, Essen

Nacke, B. Mühlbauer, A., Nikanorov A., Nauvertat, G. and Schülbe, H., (2001). Transverse Flux Heating of Metals Rolling and Treatment with reduced energy demand, Proceedings of the conference "Energy Savings in Electrical Engineering", pp. 123-126, July 2001, Publisher of Warsaw Univ. of Technology, ISBN 83-9134053-8 Warsaw

Schülbe, H.,Nikanorov,A., Nacke B. (2004) Flexible Transverse Flux Induction Heaters of Metal Strip, Proceedings of the International Symposium on Heating by Electromagnetic 
Sources. pp. 293-300, ISBN 88-86281-92-7, Padua, June 2004, Servizi Grafici Editoriali, Padua

Tudorache, T. \& Fireteanu, V (1998). 3D Numerical Modelling of New Structures for Transverse Flux Heating of Metallic Sheets,, Proceedings of the International Heating Seminar HIS 1998 pp. 117-123, 88-86281, Padua, May 1998, Servizi Grafici Editoriali, Padua 


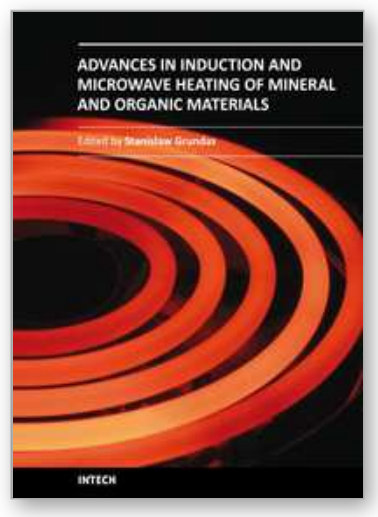

\section{Advances in Induction and Microwave Heating of Mineral and Organic Materials}

Edited by Prof. StanisÅ,aw Grundas

ISBN 978-953-307-522-8

Hard cover, 752 pages

Publisher InTech

Published online 14, February, 2011

Published in print edition February, 2011

The book offers comprehensive coverage of the broad range of scientific knowledge in the fields of advances in induction and microwave heating of mineral and organic materials. Beginning with industry application in many areas of practical application to mineral materials and ending with raw materials of agriculture origin the authors, specialists in different scientific area, present their results in the two sections: Section 1-Induction and Microwave Heating of Mineral Materials, and Section 2-Microwave Heating of Organic Materials.

\section{How to reference}

In order to correctly reference this scholarly work, feel free to copy and paste the following:

Jerzy Barglik (2011). Induction Heating of Thin Strips in Transverse Flux Magnetic Field, Advances in Induction and Microwave Heating of Mineral and Organic Materials, Prof. StanisÅ,aw Grundas (Ed.), ISBN: 978-953-307522-8, InTech, Available from: http://www.intechopen.com/books/advances-in-induction-and-microwaveheating-of-mineral-and-organic-materials/induction-heating-of-thin-strips-in-transverse-flux-magnetic-field

\section{INTECH}

open science | open minds

\author{
InTech Europe \\ University Campus STeP Ri \\ Slavka Krautzeka 83/A \\ 51000 Rijeka, Croatia \\ Phone: +385 (51) 770447 \\ Fax: +385 (51) 686166 \\ www.intechopen.com
}

\author{
InTech China \\ Unit 405, Office Block, Hotel Equatorial Shanghai \\ No.65, Yan An Road (West), Shanghai, 200040, China \\ 中国上海市延安西路65号上海国际贵都大饭店办公楼 405 单元 \\ Phone: +86-21-62489820 \\ Fax: $+86-21-62489821$
}


(C) 2011 The Author(s). Licensee IntechOpen. This chapter is distributed under the terms of the Creative Commons Attribution-NonCommercialShareAlike-3.0 License, which permits use, distribution and reproduction for non-commercial purposes, provided the original is properly cited and derivative works building on this content are distributed under the same license. 